\title{
Structural and functional analyses of the mammalian TIN2-TPP1-TRF2 telomeric complex
}

\begin{abstract}
Chunyi Hu ${ }^{1, *}$, Rekha Rai ${ }^{2, *}$, Chenhui Huang ${ }^{3,4}$, Cayla Broton ${ }^{2}$, Juanjuan Long ${ }^{1}$, Ying Xu ${ }^{1}$, Jing Xue ${ }^{1}$, Ming Lei ${ }^{3,4,5}$, Sandy Chang ${ }^{2,6,7}$, Yong Chen ${ }^{1,8}$

${ }^{I}$ State Key Laboratory of Molecular Biology, National Center for Protein Science Shanghai, Shanghai Science Research Center, CAS Center for Excellence in Molecular Cell Science, Shanghai Institute of Biochemistry and Cell Biology, Chinese Academy of Sciences; University of Chinese Academy of Sciences, 333 Haike Road, Shanghai 201210, China; ${ }^{2}$ Department of Laboratory Medicine, Yale University School of Medicine, 330 Cedar St., New Haven, CT 06510 USA; ${ }^{3}$ Ninth People's Hospital, Shanghai Jiao Tong University School of Medicine, Shanghai 200011, China, ${ }^{4}$ Shanghai Institute of Precision Medicine, Shanghai 200125, China; ${ }^{5}$ Key laboratory of Cell Differentiation and Apoptosis of Chinese Ministry of Education, Shanghai Jiao Tong University School of Medicine, Shanghai 200025, China; ${ }^{6}$ Department of Pathology, Yale University School of Medicine, 330 Cedar St., New Haven, CT 06510 USA, ${ }^{7}$ Department of Molecular Biophysics and Biochemistry, Yale University School of Medicine, 330 Cedar St., New Haven, CT 06510 USA; ${ }^{8}$ School of Life Science and Technology, ShanghaiTech University, 100 Haike Road, Shanghai 201210, China
\end{abstract}

Telomeres are nucleoprotein complexes that play essential roles in protecting chromosome ends. Mammalian telomeres consist of repetitive DNA sequences bound by the shelterin complex. In this complex, the POT1-TPP1 heterodimer binds to single-stranded telomeric DNAs, while TRF1 and TRF2-RAP1 interact with double-stranded telomeric DNAs. TIN2, the linchpin of this complex, simultaneously interacts with TRF1, TRF2, and TPP1 to mediate the stable assembly of the shelterin complex. However, the molecular mechanism by which TIN2 interacts with these proteins to orchestrate telomere protection remains poorly understood. Here, we report the crystal structure of the $\mathrm{N}$-terminal domain of TIN2 in complex with TIN2-binding motifs from TPP1 and TRF2, revealing how TIN2 interacts cooperatively with TPP1 and TRF2. Unexpectedly, TIN2 contains a telomeric repeat factor homology (TRFH)-like domain that functions as a protein-protein interaction platform. Structure-based mutagenesis analyses suggest that TIN2 plays an important role in maintaining the stable shelterin complex required for proper telomere end protection.

Keywords: shelterin structure; TIN2-TPP1; TIN2-TRF2; telomeres

Cell Research (2017) 27:1485-1502. doi:10.1038/cr.2017.144; published online 21 November 2017

\section{Introduction}

Telomeres are specialized nucleoprotein complexes at the ends of linear chromosomes that play important roles in maintaining genome stability and faithfully transmitting of genetic information [1]. Mammalian telomeres are composed of repetitive TTAGGG DNA sequences

*These two authors contributed equally to this work.

Correspondence: Yong Chen ${ }^{\mathrm{a}}$, Sandy Chang ${ }^{\mathrm{b}}$, Ming Lei ${ }^{\mathrm{c}}$

aE-mail: yongchen@sibcb.ac.cn

bE-mail: s.chang@yale.edu

${ }^{\mathrm{c} E}$-mail: 1eim@shsmu.edu.cn

Received 28 August 2017; revised 25 September 2017; accepted 17 October 2017; published online 21 November 2017 and are bound by a six-protein shelterin complex consisting of TRF1, TRF2, RAP1, TIN2, TPP1, and POT1 [2]. Telomere uncapping by deleting shelterin components activates inappropriate DNA damage signaling and repair pathways, leading to chromosome fusion or telomere loss $[1,3]$. TRF1 and TRF2 bind to the double-stranded (ds) DNA regions of telomeres and function as docking sites for many telomere-accessory proteins [4-6]. The POT1-TPP1 heterodimer binds to the single-stranded (ss) telomeric overhangs and participates in telomere length control by regulating telomerase recruitment and processivity [7-9]. POT1-TPP1 is also required to repress an ATR-dependent DNA damage response (DDR) at telomeres [10-15]. TIN2 occupies a central position in this complex and simultaneously interacts with TRF1, TRF2, 
and TPP1, bridging three major DNA-binding modules in a highly organized manner [16-19].

As the central hub of the shelterin complex, TIN2 plays critical roles in both telomere maintenance and end protection [17-23]. TIN2 is involved in TRF1-mediated telomere length regulation, stabilizing TRF1 through two distinct mechanisms. First, TIN2 protects TRF1 from tankyrase 1-mediated poly(ADP-ribosyl)ation, in turn stabilizing TRF1's association with telomeres [24]. Second, TIN2 competes with $\mathrm{SCF}^{\mathrm{FBX} 4}$ for TRF1 binding, thus preventing TRF1 from ubiquitin-dependent proteolysis [25]. TIN2 is also involved in telomere protection. By linking the POT1-TPP1 heterodimer on the $3^{\prime}$ ss overhang to the rest of the shelterin complex, TIN2 helps repress ATR signaling [22]. In addition, TIN2 inhibits ATM signaling at telomeres by stabilizing TRF2 on ds telomeric DNAs $[19,22]$. Thus, loss of TIN2 initiates DDRs via both pathways, highlighting the importance of TIN2 in telomere end protection.

Given that TIN2 interacts with three shelterin proteins and TIN2 deletion compromises the stability of both TRF1 and TRF2 at telomeres [17, 20, 22], it is difficult to define the precise roles of each of the TIN2-mediated interactions in telomere protection. This problem is partially solved by designing TIN2 mutants that specifically disrupt its interaction with TRF1 [19] or with TPP1 [18]. However, the importance of the TIN2-TRF2 interaction remains unclear.

With the exception of a short C-terminal TRFH-binding motif of TIN2 that has been structurally characterized [4], no structural information is available for TIN2. How TIN2 functions as a hub to bridge the ds and ss telomeric regions remains elusive. Here, we determine the structure of TIN2-TPP1-TRF2 ternary complex and demonstrate how TIN2 interacts cooperatively with TPP1 and TRF2. Interestingly, N-terminal domain of TIN2 shows a striking structural similarity to the TRFH domains of TRF1 and TRF2, suggesting that they may have a common ancestor, despite having diverged functionally during evolution. Our structural studies enable us to generate point mutations that specifically disrupt TIN2's capacity to interact with TRF1, TRF2, or TPP1. We found that the TIN2-TPP1 interaction is important to repress alternative non-homologous end joining (A-NHEJ)-mediated chromatid and sister telomere fusions, while the TIN2-TRF2 and TIN2-TRF1 interactions are required to prevent both classical-NHEJ (C-NHEJ)-mediated chromosome fusions and A-NHEJ-mediated chromatid fusions.

\section{Results}

Structure of the TIN2-TPP1-TRF2 ternary complex
Previous studies have shown that TIN2 interacts with TRF2 via two interacting modules [4]. One is the short TRFH-binding motif (TBM) (residues 256-276) at the C-terminal portion of TIN2, which interacts with both $\mathrm{TRF} 1_{\mathrm{TRFH}}$ and TRF $2_{\mathrm{TRFH}}$ (Figure 1A) [4]. The other is the N-terminal domain of TIN2 (residues 2-202), which rec-

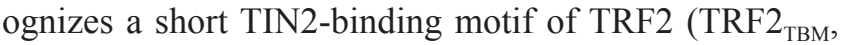
residues 350-366) (Figure 1A). Here, we used microscale thermophoresis (MST) to evaluate the contribution of each TIN2 module to the TIN2-TRF2 interaction (Supplementary information, Figure S1A). We found that TIN2 $2-202$ binds to TRF $2_{\mathrm{FL}}$ (full-length TRF2) with a $K_{\mathrm{d}}$ of $1.5 \mu \mathrm{M}$, nearly identical to the affinity of full-length TIN2 to TRF $2_{\mathrm{FL}}$ (Supplementary information, Figure S1A). In contrast, the C-terminal half of TIN2 (residues 200-355) interacts with TRF $2_{\mathrm{FL}}$ with a much lower affinity $\left(K_{\mathrm{d}} \sim 29 \mu \mathrm{M}\right)$. This result confirmed that TIN2 has two TRF2-binding modules and the $\mathrm{N}$-terminal region of TIN2 is the dominant binding site for TRF2 $2_{\mathrm{FL}}$. Next, we explored the interaction between TIN2 and TPP1. The C-terminal region of TPP1 (residues 480-544) has been shown previously to bind TIN2 [21, 26]. Further mapping by yeast two-hybrid analysis revealed that a TPP1 fragment consisting of residues 510-544 was both necessary and sufficient for interaction with TIN2 (Supplementary information, Figure S1B). Hereafter, we will refer to TPP $1_{510-544}$ as the TIN2-binding motif of TPP1 (TPP $\left.1_{\text {твM }}\right)$ (Figure 1A).

We found that TIN $2_{2-202}$ can bind to both TPP $1_{\text {твм }}$ and $\mathrm{TRF} 2_{\text {твм }}$ simultaneously, forming a stable ternary complex (Supplementary information, Figure S1C). After extensive screenings, we successfully crystallized a complex containing all three proteins $\left(\mathrm{TIN} 2_{2-202}, \mathrm{TPP} 1_{\text {твм }}\right.$, and TRF $2_{\text {твм }}$, Figure 1A) and determined its structure at a resolution of $2.2 \AA$ by single-wavelength anomalous dispersion with selenomethionine-substituted crystals (Table 1). This structure was refined to an $R$-value of $19.5 \%\left(R_{\text {free }} 22.7 \%\right)$ with good geometry. The electron density map unambiguously allowed us to trace the majority of the complex (Supplementary information, Figure S2A and S2B).

TIN $2_{2-202}$ adopts a compact fold with nine $\alpha$-helices tightly packed together with linear dimensions of $\sim 60 \AA$ $\times 40 \AA \times 30 \AA$ (Figure 1B). Residues 91-96 of TIN2 are not modeled in the structure due to their poor electron density, which is consistent with the observation that this short fragment of TIN2 is variable in size across species (Supplementary information, Figure S2C). Each TIN2 $2_{2-202}$ molecule binds to one TRF $2_{\mathrm{TBM}}$ and to one TPP $1_{\mathrm{TBM}}$ using distinct surfaces (Figure 1B). TIN2 $2-202$ can be roughly divided into two helix bundles. Helices $\alpha 3, \alpha 4, \alpha 5$, $\alpha 6$, and the N-terminal half of $\alpha 7$ ( $\alpha 7 \mathrm{a})$ of TIN2 $2_{2-202}$ form 
A

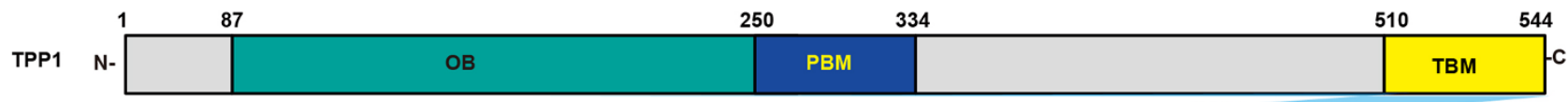

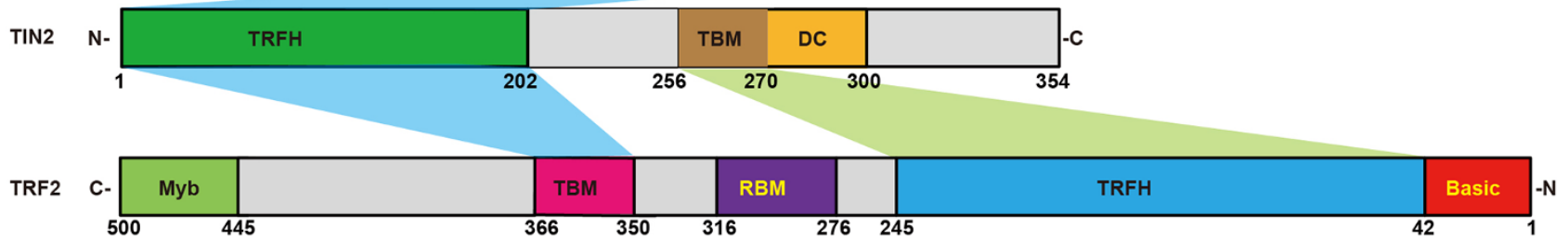

B

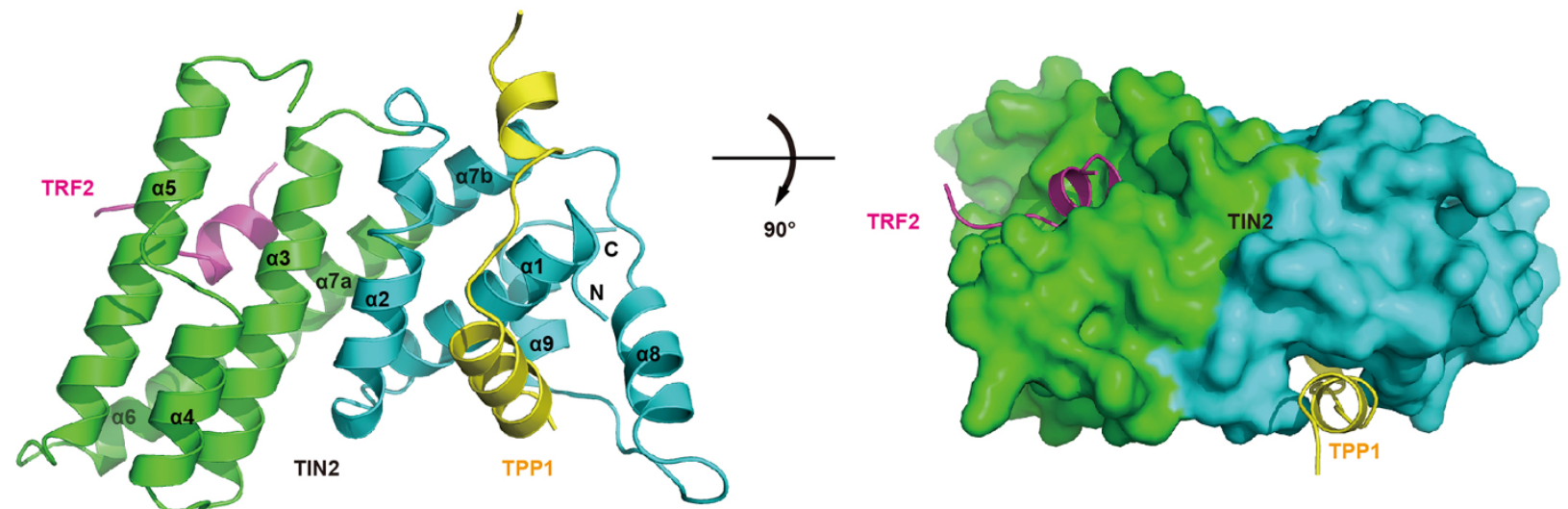

C
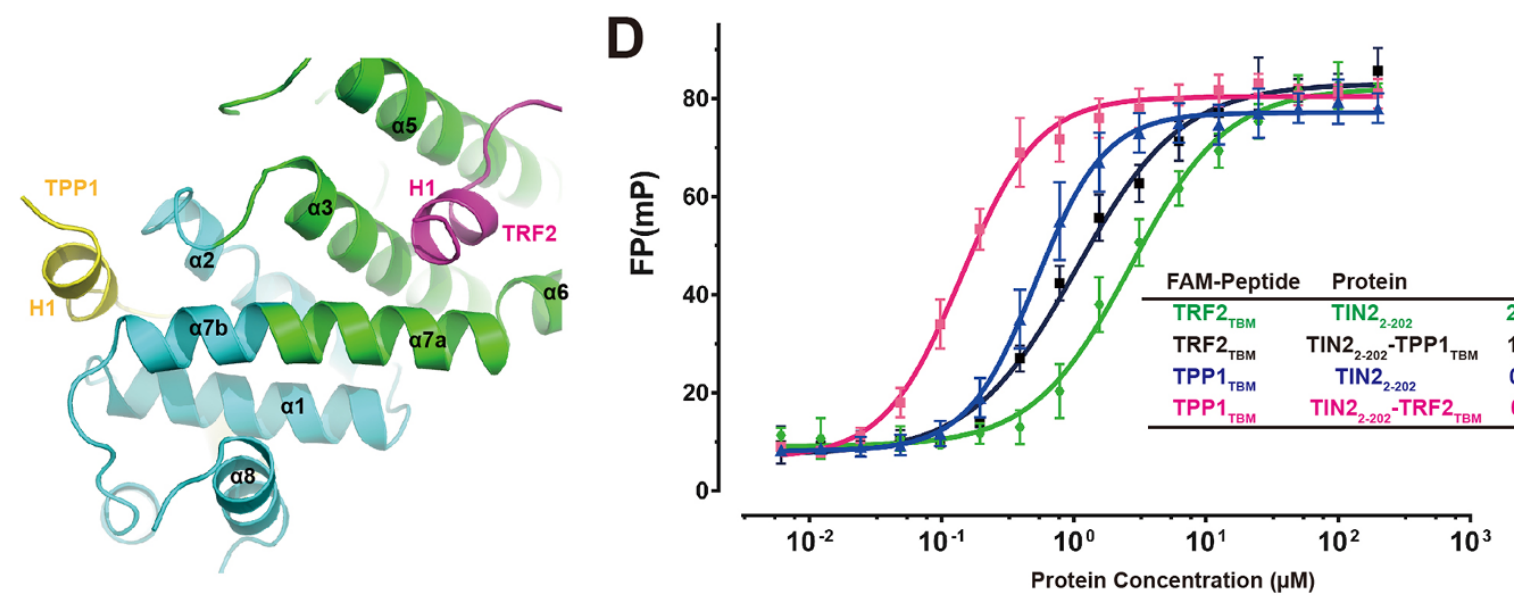

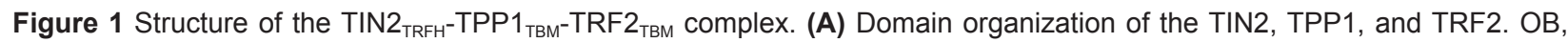
oligosaccharide-binding fold; PBM in TPP1, POT1-binding motif; TBM in TPP1, TIN2-binding motif; TBM in TIN2, TRFH-binding motif; DC, dyskeratosis congenita hotspot; Basic, an N-terminal basic-residue-rich domain; TRFH, telomeric repeat factor homology domain; RBM in TRF2, RAP1-binding motif; TBM in TRF2, TIN2-binding motif; Myb, a C-terminal DNA-binding domain. (B) Two orthogonal views of the overall structure of the TIN2 TRFH $-\mathrm{TPP} 1_{\mathrm{TBM}}-\mathrm{TRF} 2_{\mathrm{TBM}}$ complex. The left panel is shown in cartoon diagram, and TIN2 ${ }_{\text {TRFH }}$ in the right panel is shown in surface representation. TIN2 $2_{\text {TRFH }}$ is colored in green (helical bundle involved in TRF2 binding) and cyan (helical bundle involved in TPP1 binding). TPP $1_{\text {ТВM }}$ and TRF $2_{\text {TBM }}$ are colored in yellow and magenta, respectively. (C) The brace helix $\alpha 7$ mediates cooperative binding with TPP $1_{\text {TBM }}$ and TRF $2_{\text {TBM. }}$ (D) TPP $1_{\text {TBM }}$ promotes TRF2 $2_{\mathrm{TBM}}$ interaction with TIN2 $2_{\mathrm{TRFH}}$, and TRF2 ${ }_{\mathrm{TBM}}$ also enhances TPP $1_{\mathrm{TBM}}$ interaction with TIN2 $2_{\mathrm{TRFH}}$, as shown by fluorescence polarization assays. 
Table 1 Data collection, phasing, and refinement statistics for SAD (SeMet) TIN $2_{\mathrm{TRFH}^{-}}-\mathrm{TPP} 1_{\mathrm{TBM}}-\mathrm{TRF} 2_{\mathrm{TBM}}$ structure

\begin{tabular}{|c|c|}
\hline & SeMet TIN $2_{\mathrm{TRFH}^{-}}-\mathrm{TPP} 1_{\mathrm{TBM}}-\mathrm{TRF} 2_{\mathrm{TBM}}$ \\
\hline \multicolumn{2}{|l|}{ Data collection } \\
\hline Space group & $C 222_{1}$ \\
\hline \multicolumn{2}{|l|}{ Cell dimensions } \\
\hline $\mathrm{a}, \mathrm{b}, \mathrm{c}(\AA)$ & $46.222,112.258,121.209$ \\
\hline \multirow[t]{2}{*}{$\alpha, \beta, \gamma\left(^{\circ}\right)$} & $90,90,90$ \\
\hline & Se Peak \\
\hline Wavelength $(\AA)$ & 0.97854 \\
\hline Resolution $(\AA)$ & $50-2.2$ \\
\hline$R_{\text {merge }}$ & $0.101(0.472)^{*}$ \\
\hline$I / \sigma I$ & $14.3(2.1)$ \\
\hline Completeness $(\%)$ & $99.8(99.7)$ \\
\hline Redundancy & $3.4(3.3)$ \\
\hline \multicolumn{2}{|l|}{ Refinement } \\
\hline Resolution $(\AA)$ & $32.8-2.2$ \\
\hline No. of reflections & 16341 \\
\hline$R_{\text {work }} / R_{\text {free }}(\%)$ & $19.5 / 22.7$ \\
\hline \multicolumn{2}{|l|}{ No. of atoms } \\
\hline TIN2 & 1539 \\
\hline TPP1 & 195 \\
\hline TRF2 & 101 \\
\hline Water & 72 \\
\hline \multicolumn{2}{|l|}{ B-factors $\left(\AA^{2}\right)$} \\
\hline TIN2 & 47.7 \\
\hline TPP1 & 57.9 \\
\hline TRF2 & 63.7 \\
\hline Water & 50.5 \\
\hline \multicolumn{2}{|l|}{ RMS deviations } \\
\hline Bond lengths ( $\AA$ ) & 0.004 \\
\hline Bond angles $\left(^{\circ}\right)$ & 0.600 \\
\hline
\end{tabular}

*Values in parentheses are for the highest-resolution shell.

the first helical bundle, packing against TRF $2_{\mathrm{TBM}}$ (Figure 1B). The other is comprised of helices $\alpha 1, \alpha 2, \alpha 8, \alpha 9$, and the C-terminal half of $\alpha 7(\alpha 7 b)$ and this helical bundle interacts with ТРP1 $1_{\text {твм }}$ (Figure 1B).

Previous studies revealed that TPP 1 promotes the interaction between TIN2 and TRF2 [21]. The ternary complex structure shown here suggests a molecular mechanism for this cooperativity. Although ТРP $1_{\text {твм }}$ and TRF $2_{\text {TBM }}$ bind to two different pockets in $\mathrm{TIN} 2_{2-202}$, the long helix 7 of $\mathrm{TIN} 2_{2-202}$ contacts both TPP $1_{\mathrm{TBM}}$ and TRF $2_{\text {твм }}$ simultaneously (Figure 1C). This $\alpha 7$ helix functions like a seesaw, with its two ends seated by $\mathrm{TPP} 1_{\mathrm{TBM}}$ and TRF $2_{\text {твм }}$, respectively (Figure 1C). We postulate that the binding of TPP $1_{\text {твM }}$ to TIN2 $2_{2-202}$ will allosterically regulate the $\alpha 7$ helix of TIN2 to maintain an optimal configuration to bind to TRF $2_{\mathrm{TBM}}$, and vice versa. In support of this notion, fluorescence polarization assays confirmed that the binding affinity between TIN $2_{2-202}-\mathrm{TPP} 1_{\mathrm{TBM}}$ and TRF $2_{\text {твм }}$ is $\sim 2.6$-fold higher than that between TIN2 $2_{2-202}$ and TRF $2_{\text {твм }}$ (Figure 1D), indicating that TPP $1_{\mathrm{TBM}}$ can enhance the interaction between TIN2 $2_{2-202}$ and TRF2 $2_{\text {TBM }}$. Similarly, TRF $2_{\text {TвM }}$ binding to TIN $2_{2-202}$ also increased the binding affinity between TIN $2_{2-202}$ and TPP $1_{\text {твм }}$ by 3.7-fold (Figure 1D). Taken together, these data suggest that TIN2 $2-202$ cooperatively interacts with TPP1 and TRF2 to ensure the stable assembly of the shelterin complex.

TIN2 2-202 structurally resembles the TRFH domains of TRF1 and TRF2

Next, we performed an unbiased search for structurally homologous proteins to the TIN $2_{2-202}$ domain using DALI [27]. Surprisingly, we found that TIN $2_{2-202}$ is closely related to the TRFH domains of TRF1 and TRF2 (Figure 2A and 2B). TIN $2_{2-202}$ can be superimposed onto the TRF $1_{\mathrm{TRFH}}$ and TRF $2_{\mathrm{TRFH}}$ domains with root-meansquare deviation (rmsd) values of 3.5 and $3.4 \AA$ for 123 and 131 equivalent $C \alpha$ pairs, respectively, although structure-based sequence alignment shows $<9 \%$ identity between TIN2 $2_{2-202}$ and TRFH domains (Figure 2C). Superimposition of $\mathrm{TIN} 2_{2-202}$ with $\mathrm{TRF} 1_{\mathrm{TRFH}}$ and $\mathrm{TRF} 2_{\mathrm{TRFH}}$ clearly reveals that the first seven $\alpha$-helices of the TIN $2_{2}$ 202 structurally resemble the $\alpha 3-\alpha 9$ helices in TRF $1 / 2_{\text {TRFH }}$ (Figure 2A and 2C). In addition, both $\mathrm{TIN} 2_{2-202}$ and the TRFH domains of TRF1 and TRF2 function as protein-interaction platforms that utilize a similar surface to interact with their respective binding partners - TPP1 for TIN2 $2_{2-202}$ and TBM-containing proteins for $\mathrm{TRF} 1_{\mathrm{TRFH}}$ and TRF $2_{\mathrm{TRFH}}$ (Figure 2B). Together, these structural and functional similarities suggest that $\mathrm{TIN} 2_{2-202}$ and the TRFH domains of TRF1 and TRF2 are very likely to be evolutionarily related. Therefore, we name $\mathrm{TIN} 2_{2-202}$ as the TRFH domain of TIN2 (TIN2 ${ }_{\mathrm{TRFH}}$ ) (Figure 1A).

\section{The TIN2-TPP1 interface}

In the ternary complex, each $\mathrm{TPP} 1_{\mathrm{TBM}}$ polypeptide is folded into a helix-loop-helix motif $\left(\mathrm{H} 1-\mathrm{L}_{12}-\mathrm{H} 2\right)$ (Figure $1 \mathrm{~B})$. Both helices and the connecting loop make extensive contacts with TIN $2_{\text {TRFH }}$, burying $\sim 1268 \AA^{2}$ of surface area at their interface (Figure 1B). The driving force for the binding of TPP $1_{\mathrm{TBM}}$ to TIN2 $2_{\mathrm{TRFH}}$ is van der Waals interactions, as most conserved residues of TPP $1_{\mathrm{TBM}}$ are hydrophobic in nature (Supplementary information, Figure S2D). This observation is consistent with the finding that ТРP $1_{\text {Твм }}$ cannot be purified by itself and must be 
A
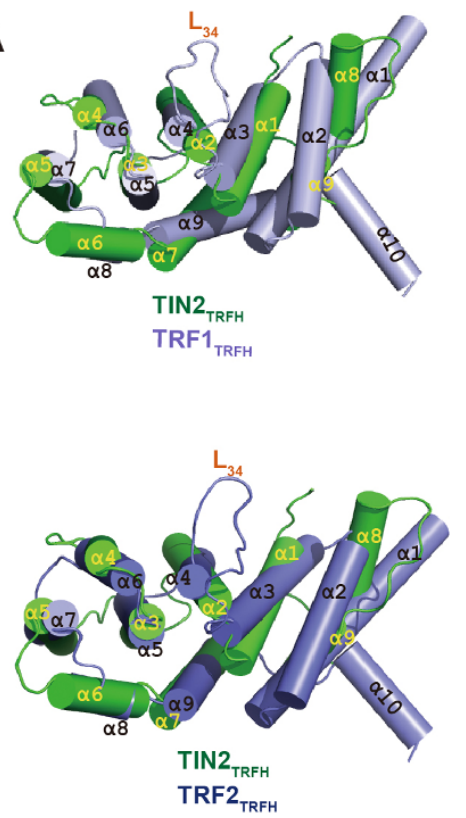
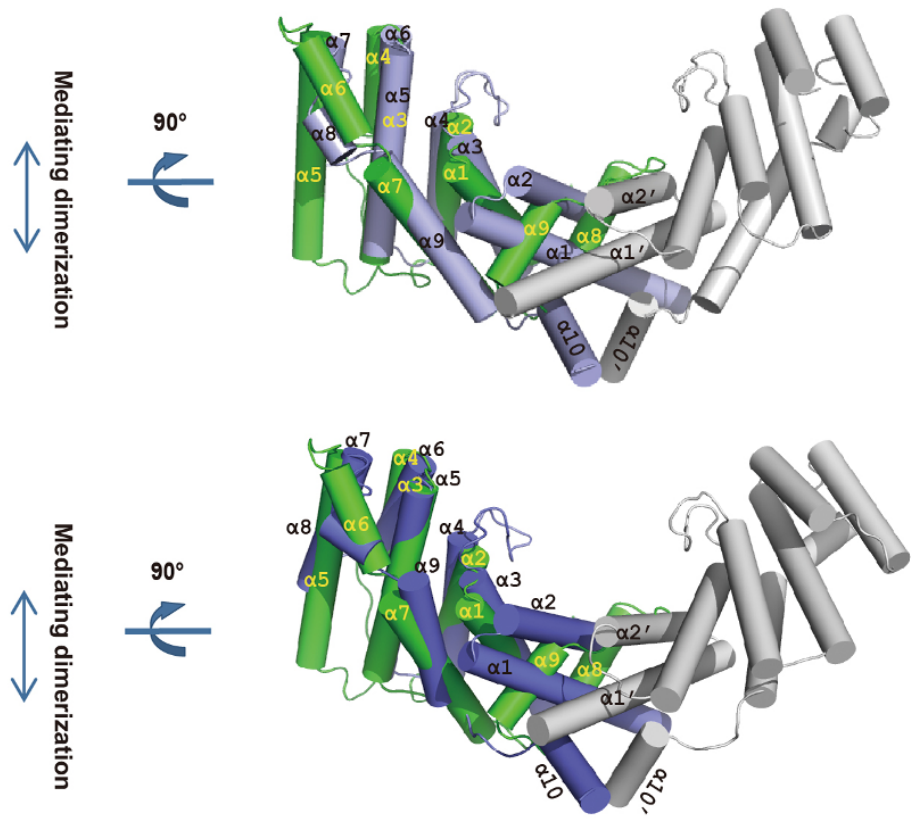

B
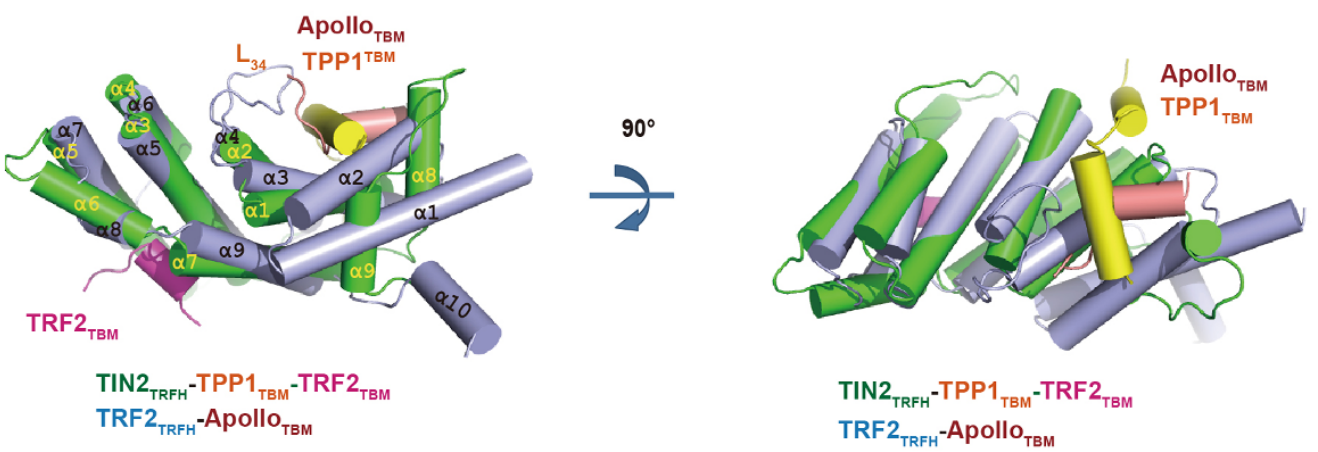

C

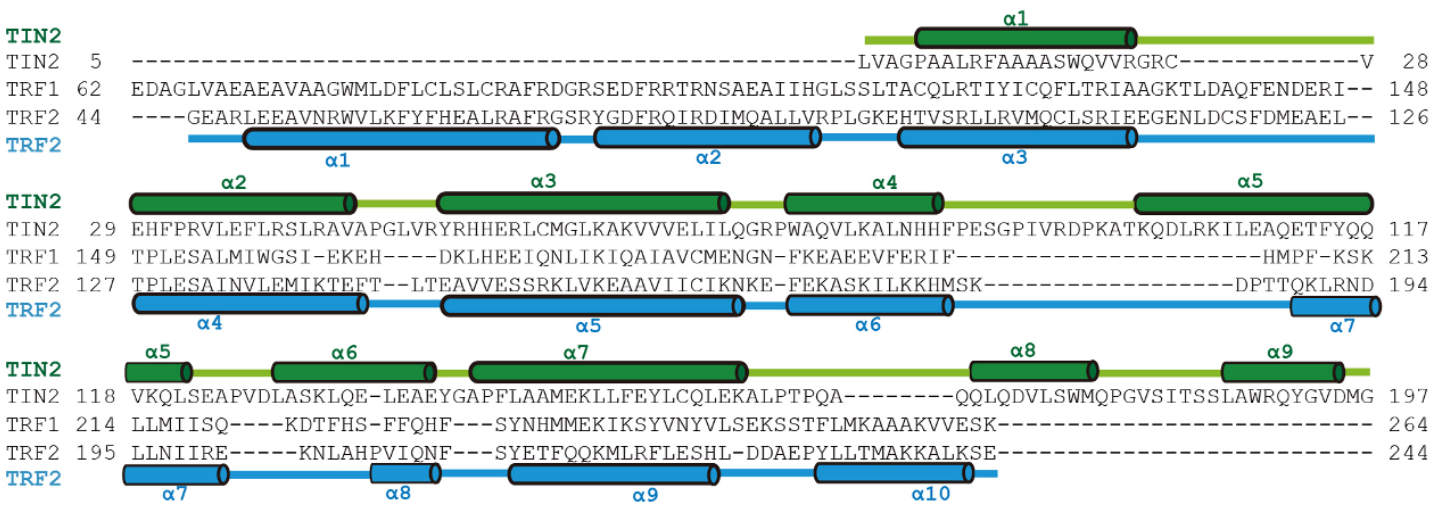

Figure 2 TIN2 $2_{\text {TRFH }}$ contains a TRFH-like fold. (A) Superimposition of TIN2 $2_{\text {TRFH }}$ (green), TRF $1_{\text {TRFH }}$ (light blue) and TRF2 $2_{\text {TRFH }}$ (blue) shown in two orthogonal views. The left panel shows monomeric TRF $1 / 2_{\text {TRFH }}$ and the right panel shows the dimeric form of TRF $1 / 2_{\text {TRFH }}$ with one monomer colored in gray. TIN2 $2_{\text {TRFH }} \alpha 8$ and $\alpha 9$ disrupt the dimerization interface formed by $\alpha 1, \alpha 2$,

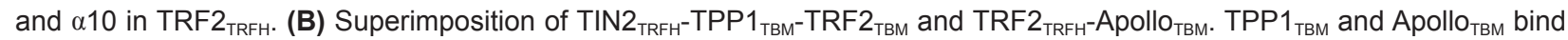
to the similar surface pockets on TIN2 $2_{\text {TRFH }}$ and TRF2 $2_{\text {TRFH }}$, respectively. (C) Structure-based sequence alignment of TIN2 $2_{\text {TRFH }}$, TRF1 $1_{\mathrm{TRFH}}$, and TRF2 ${ }_{\mathrm{TRFH}}$. Secondary structure assignments based on the TIN2 $2_{\mathrm{TRFH}}$ and TRF2 $2_{\mathrm{TRFH}}$ structures are shown as cylinders ( $\alpha$-helices) and lines (loops). 
A

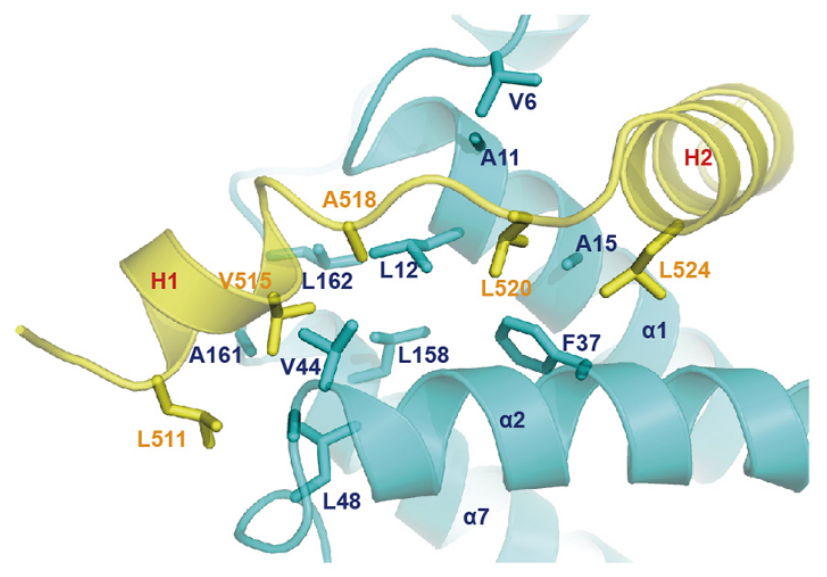

C

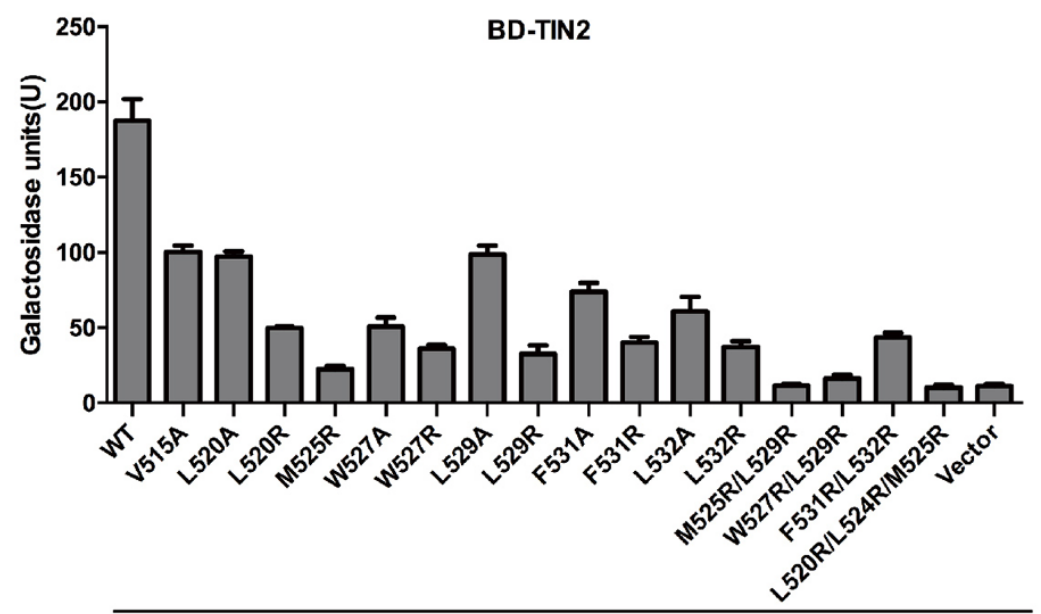

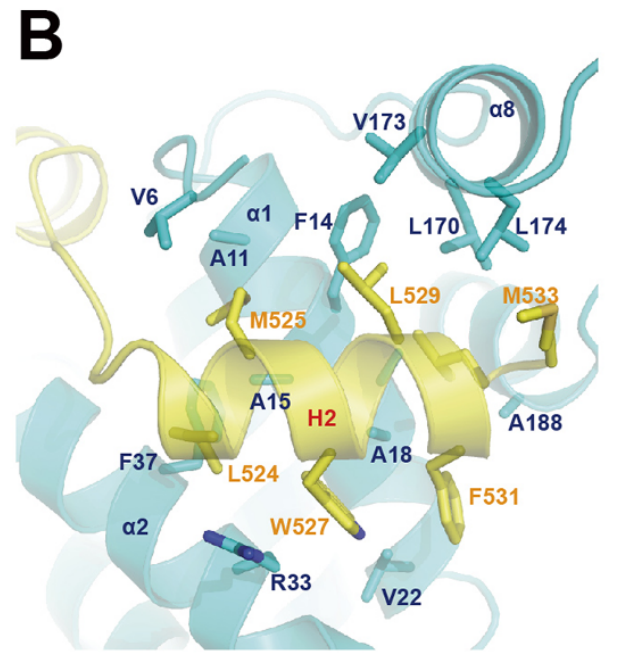

D

AD-TPP1

Figure 3 The interface between TIN2 $2_{\text {TRFH }}$ and TPP $1_{\text {TBM }}$ (A) Details of hydrophobic interactions around $\mathrm{H} 1$ and $\mathrm{L}_{12}$ of TPP1. The interaction residues are presented as ball-and-stick models. TPP1 residues are colored in yellow and TIN2 residues are colored in cyan. (B) Details of hydrophobic contacts between H2 of TPP1 and TIN2. (C, D) Effects of mutations in the TPP1 $_{\text {TBM }}\left(\right.$ C) and TIN2 $2_{\text {TRFH }}$ (D) domains on the interaction between TIN2 and TPP1 analyzed in a yeast two-hybrid assay. Data are the average of three independent $\beta$-galactosidase measurements.

coexpressed and copurified along with TIN $2_{\mathrm{TRFH}}$. The $\mathrm{H} 1$ and $\mathrm{H} 2$ helices of $\mathrm{TPP} 1_{\text {Твм }}$ pack against the floors of two hydrophobic grooves on TIN $2_{\mathrm{TRFH}}$, separated by helices $\alpha 1$ and $\alpha 2$ of TIN2 (Figure 3A and 3B). In addition, the loop connecting helices $\mathrm{H} 1$ and $\mathrm{H} 2$ of $\mathrm{TPP} 1_{\text {ТвM }}$ also makes intimate contacts with TIN2 $2_{\text {TRFH }}$ (Figure $3 \mathrm{~A}$ ). The core of this extended interface between ТPР $1_{\text {Твм }}$ and TIN $2_{\text {TRFH }}$ consists of a panel of hydrophobic residues from both proteins, including V515, L520, L524, M525, W527, and L529 of TPP $1_{\text {твм }}$, and V6, L12, A15, A18, F37, L158, and L162 of TIN2 ${ }_{\mathrm{TRFH}}$ (Figure $3 \mathrm{~A}$ and 3B). The extensive contacts among the side chains of these residues mediate the specificity of $\mathrm{TPP} 1_{\mathrm{TBM}}$ recognition by TIN $2_{\text {TRFH }}$.

To validate the TIN2-TPP1 interface observed in the crystal structure, we mutated specific residues involved in the TIN2-TPP1 interaction and examined their binding using a yeast two-hybrid assay. Consistent with the structural data, mutations of single residues from helices H1, $\mathrm{H} 2$, or loop $\mathrm{L}_{12}$ of TPP $1_{\text {твм }}$ destabilized the TPP1-TIN2 interaction, suggesting that all three modules contribute to TIN2-TPP1 interaction (Figure 3C). In particular, mutations on the $\mathrm{H} 2$ helix of $\mathrm{TPP} 1_{\mathrm{TBM}}$ conferred the most disrupting effects, suggesting that helix $\mathrm{H} 2$ is the most 
important region responsible for binding with TIN2 (Figure 3C). Similarly, hydrophobic residues on the TIN2 interface are also required for binding to TPP1. Substitution of TIN $2^{\mathrm{A} 15}$ with a positively charged, bulky arginine residue completely disrupted the TIN2-TPP1 interaction (Figure 3D). In contrast, alanine substitution of TIN2 interface residues did not alter the hydrophobicity of the binding pocket for TPP $1_{\mathrm{TBM}}$, and thus only partially impeded the interaction (Figure 3D). Taken together, we conclude that the hydrophobic contacts observed in the crystal structure are the major biophysical driving force for the interaction between TIN2 and TPP1.

\section{The TIN2-TRF2 interface}

The interaction between TIN $2_{\mathrm{TRFH}}$ and TRF $2_{\mathrm{TBM}}$ is mediated by a combination of van der Waals contacts and electrostatic interactions, burying $\sim 740 \AA^{2}$ of surface area that corresponds to $47 \%$ of the total surface area of TRF $2_{\text {TBM. }}$. Close inspection of the TIN $2_{\mathrm{TRFH}}-\mathrm{TRF} 2_{\mathrm{TBM}}$ interface reveals three adjacent binding modules (Figure 4A; Supplementary information, Figure S2E). The most prominent contribution to the TIN2-TRF2 interface is from the central helix of TRF $2_{\text {Твм }}\left({ }_{359}\right.$ ISRLVL $\left._{364}\right)$ (Figure 4A). Three hydrophobic residues (TRF2 $2^{\mathrm{I359}}, \mathrm{TRF} 2^{\mathrm{L} 362}$, and $\mathrm{TRF}^{\mathrm{V} 363}$ ) in the center of this helix form a hydrophobic core that fits snugly into a large triangular-shaped pocket formed by helices $\alpha 3, \alpha 5$, and $\alpha 7$ of TIN $2_{\text {TRFH }}$ (Figure 4B). The side chains of each of these residues make intimate contacts with a panel of hydrophobic amino acids at the corresponding vertices in the triangular pocket of TIN2 $2_{\text {TRFH }}$ (Figure 4B). Consistent with their importance for binding, arginine substitution of any of these residues severely impaired the interaction between TRF2 and TIN2, while substitutions with nonpolar alanine residue only modestly weakened this interaction (Figure 4E). Yeast two-hybrid analysis also revealed that TIN2 mutations on the other side of the interface (F87A, G60R, and A110R) completely disrupted the TIN2-TRF2 interaction (Figure 4B and 4F), further underscoring the importance of the hydrophobic contacts for the interaction between TRF $2_{\text {TBM }}$ and TIN $2_{\mathrm{TRFH}}$.

In addition to these hydrophobic contacts, the backbone carbonyls of TRF2 $2^{\mathrm{L} 362}$ and TRF2 ${ }^{\mathrm{V} 363}$ accept two hydrogen bonds from the side chain of $\mathrm{TIN} 2^{\mathrm{R} 56}$ (Figure 4C), while the backbone amide and side-chain hydroxyl group of TRF $2^{\mathrm{S} 360}$ donate two hydrogen bonds to TIN2 ${ }^{\mathrm{Y} 139}$ and $\mathrm{TIN}^{\mathrm{E} 138}$ (Figure 4D). These hydrogen-bonding interactions further stabilize interactions between the TRF $2_{\text {TBM }}$ helix and TIN $2_{\mathrm{TRFH}}$. Moreover, both the $\mathrm{N}$ - and $\mathrm{C}$-termini of TRF $2_{\text {твм }}$ contribute to the binding to TIN2 via electrostatic interactions. They function as two arms that facilitate the docking of the central helix of TRF $2_{\text {твм }}$ onto TIN2 (Figure 4A). The C-terminal tail of TRF $2_{\text {TBM }}$ contains two glutamate residues $\left({ }_{365} \mathrm{EE}_{366}\right)$, which extend into a basic patch of TIN2 (Figure 4A). TRF2 ${ }^{\mathrm{E} 365}$ forms an intermolecular salt bridge with $\mathrm{TIN} 2^{\mathrm{K} 106}$ and an intramolecular salt bridge with TRF2 ${ }^{\mathrm{R} 361}$ (Figure 4C). TRF2 ${ }^{\mathrm{E} 366}$ mediates two electrostatic contacts with $\mathrm{TIN} 2^{\mathrm{R} 56}$ and $\mathrm{TIN} 2^{\mathrm{R} 52}$ (Figure $4 \mathrm{C}$ ). In sharp contrast, the $\mathrm{N}$-terminus of TRF $2_{\text {Tвм }}\left({ }_{355} \mathrm{KRMT}_{358}\right)$ is basic in nature and sits on an acidic surface of TIN2 (Figure 4A). TR$\mathrm{F} 2^{\mathrm{R} 356}$ and $\mathrm{TRF} 2^{\mathrm{K} 355}$ form three salt bridges with TIN2 $2^{\mathrm{E} 109}$ and TIN2 ${ }^{\mathrm{E} 138}$ (Figure 4D). Charge-swapping mutations of these residues impaired the binding of TRF2 to TIN2, underscoring the importance of these electrostatic interactions to the TIN2 $2_{\mathrm{TRFH}}-\mathrm{TRF} 2_{\mathrm{TBM}}$ interface (Figure 4E). Interestingly, a TRF2 ${ }^{\mathrm{R} 356 \mathrm{C}}$ mutation has been identified in lung adenocarcinoma [28] and a TIN $2^{\mathrm{K} 106 \mathrm{~N}}$ mutation has been found in neuroendocrine tumors [29], suggesting that mutations disrupting the TRF2-TIN2 interaction may be cancer-promoting.

\section{Mutational analyses of TIN2-mediated interactions}

To extend the results from our structural studies, we examined whether the TIN2 mutations disrupted the interactions with TRF1, TRF2, and TPP1 in vivo. We generated specific point mutations in TIN2 identified by our structural studies to disrupt its binding to only one interaction partner: A15R to disrupt the TIN2-TPP1 interaction, L260E to disrupt the TIN2-TRF1 interaction, and G60R or A110R to disrupt the TIN2-TRF2 interaction. Co-immunoprecipitation (Co-IP) analysis revealed that the A15R mutation completely abolished the interaction between TIN2 and TPP1 while not affecting TIN2TRF2 and TIN2-TRF1 interactions (Figure 5A). The L260E mutation specifically abolished the interaction of TIN2 with Myc-TRF1 but not with TPP1 or TRF2 (Figure 5B). Notably, the G60R and A110R mutations of TIN2 severely reduced the interaction with TRF2, but did not eliminate it (Figure 5C). Combining the TIN2 mutation L260E with G60R or A110R completely disrupted the interaction with TRF2 (Figure 5C). This result indicates that the secondary TRF2-binding modules of TIN2 (TIN2 $2_{\text {TBM }}$, residues 256-276) can mediate a weak interaction with TRF2 when the TIN2 $2_{\text {TRFH }}-$ TRF $2_{\text {TBM }}$ interaction is disrupted (Figure 1A; Supplementary information, Figure S1A), although we cannot exclude the possibility that the secondary weak-binding site of TIN2 (TIN2 $2_{\text {TвM }}$ ) may indirectly interact with TRF2. How TIN $2_{\text {TBM }}$ contributes to TRF2 binding in vivo needs further investigation.

To confirm our Co-IP results, we investigated whether the telomeric accumulation of TIN2 depends on its interactions with TRF1, TRF2, or TPP1 in vivo. We expressed shRNA-resistant WT Flag-mTIN2 or Flag-mTIN2 

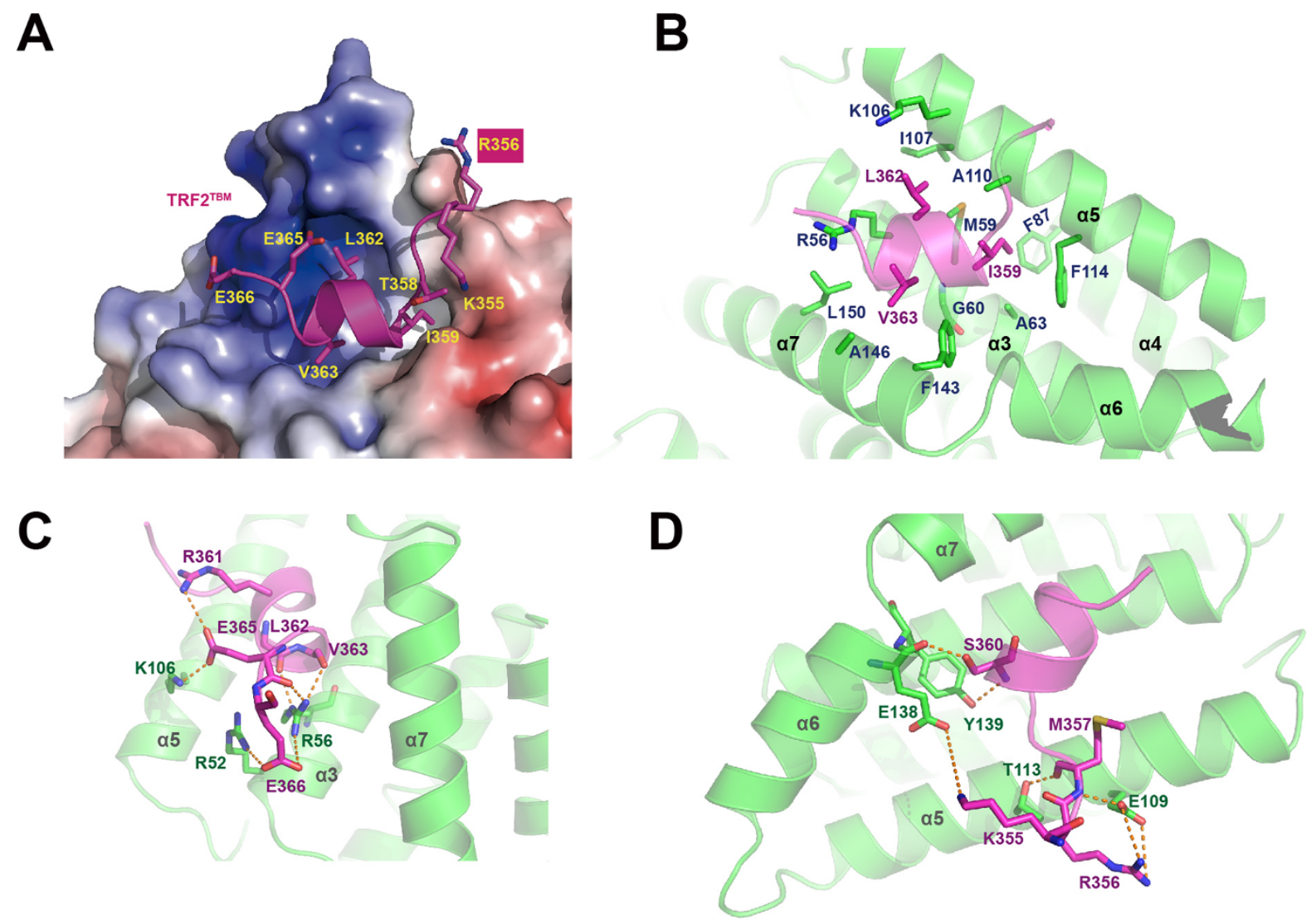

E
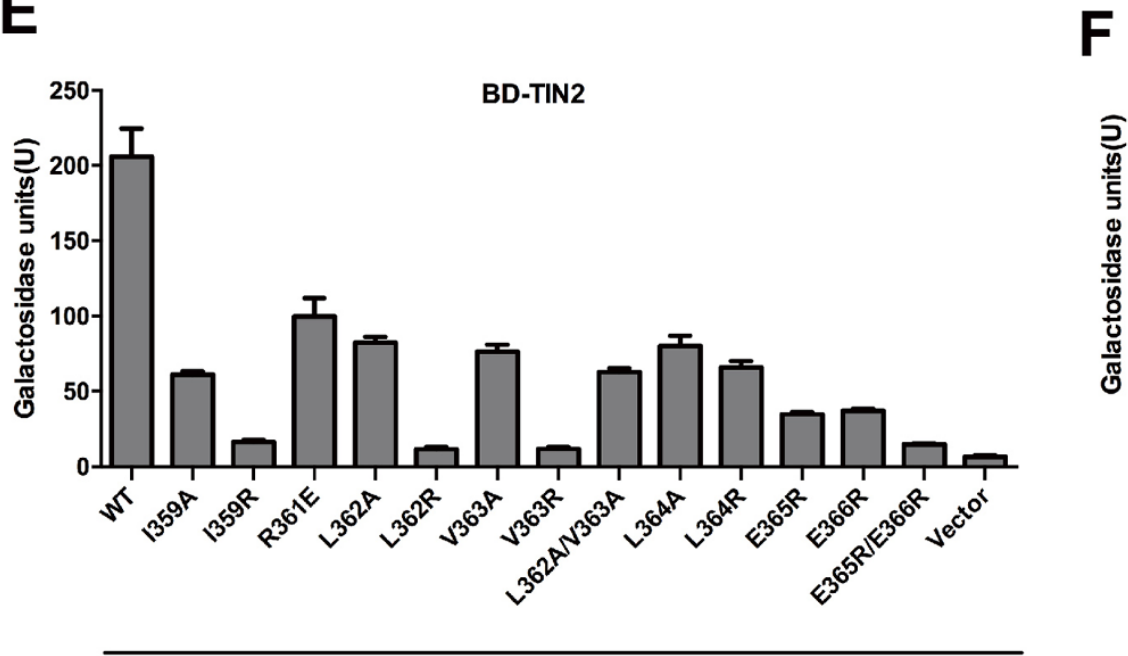

AD-TRF2

Figure 4 The interface between TIN2 $2_{\mathrm{TRFH}}$ and TRF2 $2_{\mathrm{TBM}}$. (A) TRF2 $2_{\mathrm{TBM}}$ sits in a groove of TIN2 $2_{\mathrm{TRFH}}$ shown in surface representation. TIN2 $2_{\text {TRFH }}$ is colored according to its electrostatic potential (positive potential, blue; negative potential, red). (B) Details of hydrophobic interactions in the center of TRF $2_{\text {твм. }}$ The interaction residues are presented as ball-and-stick models. TRF2 residues are colored in magenta and TIN2 residues are colored in green. (C) The C-terminal glutamate residues in TRF2 ${ }_{\text {TBM }}$ form salt bridges and hydrogen bonds with basic residues from $\alpha 3$ and $\alpha 5$ of TIN2 ${ }_{\text {TRFH. }}$ Salt bridges and hydrogen-bonding interactions are shown as orange dashed lines. (D) The positively charged residues in the N-terminal TRF2 ${ }_{\text {TBM }}$ form salt bridges and hydrogen bonds with basic residues from $\alpha 5$ and $\alpha 6$ of TIN2 ${ }_{\text {TRFH. }}$ (E, F) Effects of mutations in the TRF2 $2_{\text {TBM }}(E)$ and TIN2 ${ }_{\text {TRFH }}$ (F) domains on the interaction between TIN2 and TRF2 analyzed in a yeast two-hybrid assay. Data are the average of three independent $\beta$-galactosidase measurements. 
A
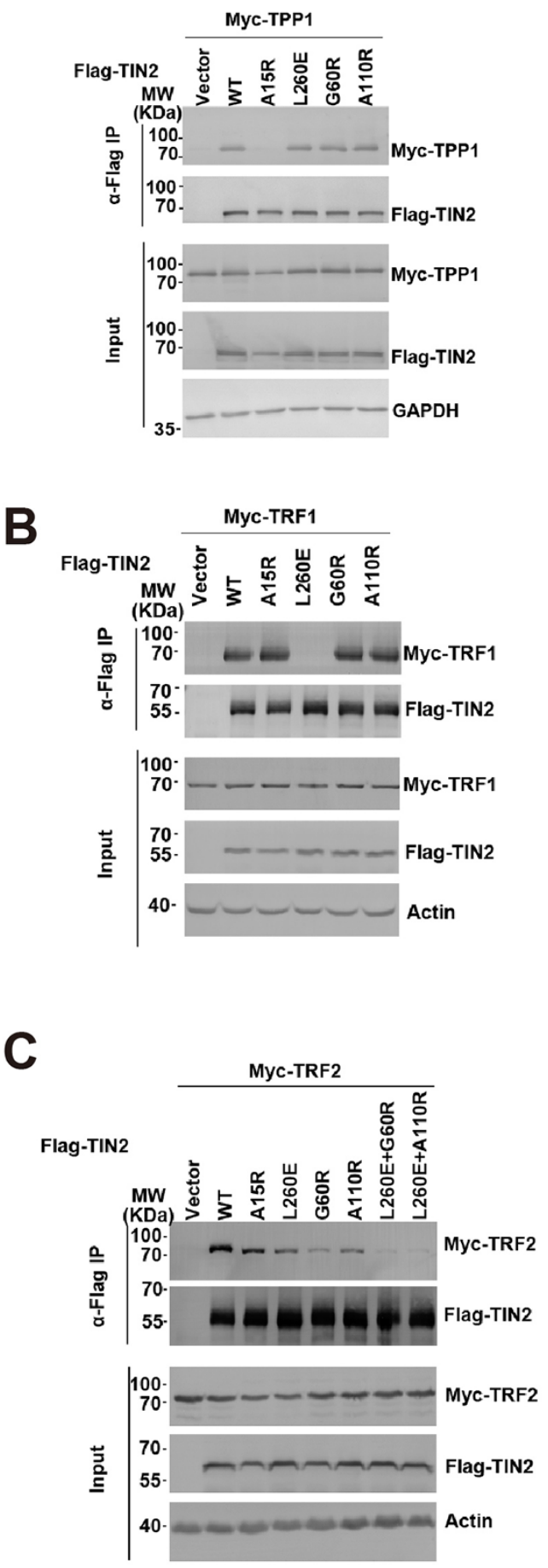

D

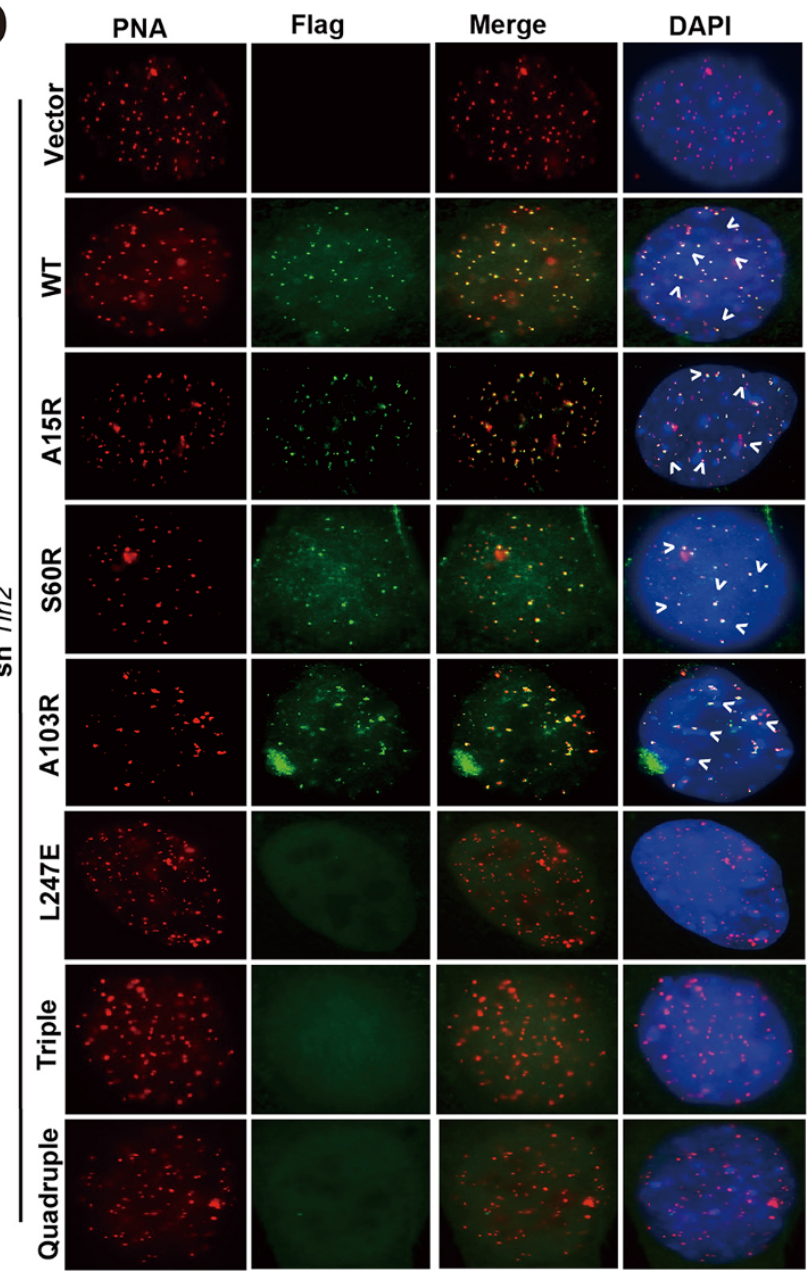

E

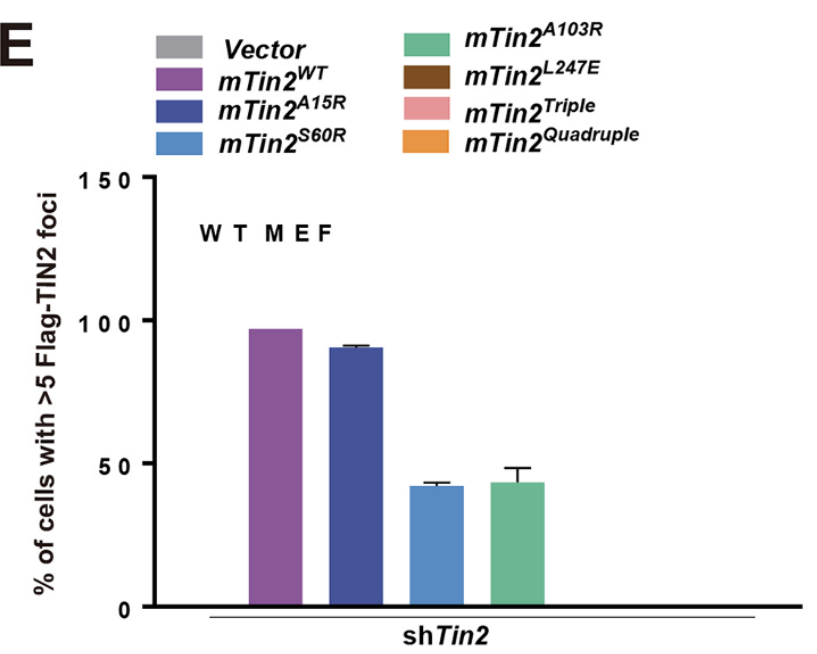

Figure 5 Analysis of TIN2 interaction with binding partners in vivo. (A-C) 293T cells expressing the indicated protein were immunoprecipitated with Flag antibody and immunoblotted with Flag and Myc antibodies. The input represents $5 \%$ of the total cell lysate used for immunoprecipitation. GADPH or actin was used as loading control. (D) WT MEFs were reconstituted with WT or indicated mTIN2 mutants before Tin2 shRNA was used to deplete endogenous TIN2. Telomeres were visualized with telomere PNA-FISH (red), anti-Flag antibody to visualize mTIN2 (green), and DAPI staining to visualize nuclei (blue). (E) Quantification of the percent of cells with $\geq 5$ foci of WT Flag mTIN2 and the mutants on telomeres in (D). Data are the mean of two independent experiments \pm SEM; more than 100 nuclei were examined per experiment. 
mutants in mouse embryo fibroblasts (MEFs) and then removed endogenous TIN2 using shRNA against mTin2 (shTin2). Immunofluorescence microscopy (IF) revealed that $\sim 90 \%$ of the cells expressing both WT Flag-mTIN2 and Flag-mTIN2 ${ }^{\mathrm{A} 15 \mathrm{R}}$ displayed $\geq 5$ TIN2-positive foci colocalized with telomeres per nucleus (Figure 5D and $5 \mathrm{E})$, suggesting that interaction with $\mathrm{mTPP} 1$ is dispensable for mTIN2's localization to telomeres. In contrast, mTIN2 ${ }^{\mathrm{S} 60 \mathrm{R}}$ (equivalent to hTIN2 ${ }^{\mathrm{G} 60 \mathrm{R}}$ ) and mTIN2 $2^{\mathrm{A} 103 \mathrm{R}}$ (equivalent to hTIN2 $2^{\mathrm{A} 110 \mathrm{R}}$ ) mutants showed telomeric accumulation only in $\sim 45 \%$ of the cells, and showed diffuse nucleoplasmic staining in the remaining cells (Figure $5 \mathrm{D}$ and $5 \mathrm{E}$ ). The $\mathrm{mTIN} 2^{\mathrm{L} 247 \mathrm{E}}$ (equivalent to $\mathrm{hTIN} 2^{\mathrm{L} 260 \mathrm{E}}$ ) mutant was completely unable to localize to telomeres (Figure 5D and 5E). We also generated triple (Flag-mTI$\mathrm{N} 2^{\mathrm{A} 15 \mathrm{R}, \mathrm{A} 103 \mathrm{R}, \mathrm{L} 247 \mathrm{E}}$ ) and quadruple (Flag-mTIN2 $2^{\mathrm{A} 15 \mathrm{R}, \mathrm{S} 60 \mathrm{R} \text {, }}$ A103R, L247E ) mutants and, as expected, neither of them localized to telomeres (Figure 5D and 5E). These data suggest that the interaction between mTIN2 and mTRF1 is absolutely essential for mTIN2 localization to telomeres, consistent with an earlier report [19]. We also examined the telomeric localization of human TIN2 mutants in human U2OS cells. Similarly, we found that Flag-TIN2 ${ }^{\mathrm{A} 15 \mathrm{R}}$ was localized to telomeres as efficiently as TIN $2^{\mathrm{WT}}$ (Supplementary information, Figure S3A and S3B). In contrast, $<10 \%$ of the cells expressing $\mathrm{TIN} 2^{\mathrm{G} 60 \mathrm{R}}$, TIN $2^{\mathrm{A} 110 \mathrm{R}}$, and TIN2 ${ }^{\mathrm{L} 260 \mathrm{E}}$ mutants showed $\geq 5$ TIN2-positive foci on telomeres (Supplementary information, Figure S3A and S3B). No colocalization of Flag-TIN2 triple and quadruple mutants with telomeres was detected (Supplementary information, Figure S3A and S3B). Taken together, these results suggest that interactions with both TRF1 and TRF2, but not TPP1, are required for efficient TIN2 localization to telomeres in human and mouse cells.

\section{TIN2 protects telomeres from engaging in DNA damage signaling and repair}

To determine whether perturbing TIN2's interaction with TRF1, TRF2, and TPP1 activates DDR at telomeres, we used the dysfunctional telomere-induced DNA damage foci (TIF) assay to monitor the recruitment of DNA damage markers $\gamma-\mathrm{H} 2 \mathrm{AX}$ and 53BP1 to telomeres bearing TIN2 mutations. While TIN2-depleted MEFs reconstituted with vector control resulted in TIF formation in $40 \%$ of MEFs examined, reconstitution with WT mTIN2 almost completely repressed the localization of $\gamma$-H2AX and 53BP1 to telomeres, with only $\sim 10 \%$ of cells displaying $\geq 5$ TIFs per nucleus (Figure $6 \mathrm{~A}$ and $6 \mathrm{~B}$; Supplementary information, Figure S4A and S4B). In sharp contrast, reconstitution with mTIN2 ${ }^{\mathrm{A} 15 \mathrm{R}}, \mathrm{mTIN}^{\mathrm{S} 60 \mathrm{R}}$, mTIN2 $2^{\mathrm{A} 103 \mathrm{R}}$, and $\mathrm{mTIN} 2^{\mathrm{L} 247 \mathrm{E}}$ all resulted in robust TIF formation, with $60 \%-80 \%$ of cells displaying $\geq 5$ TIFs per nucleus (Figure 6A and 6B; Supplementary information, Figure S4A and S4B). This two-fold increase in the number of TIFs over vector baseline levels suggests that these single TIN2 mutants exerted a dominant negative effect at telomeres (Figure 6B; Supplementary information, Figure S4B), a notion further supported by the observation that increased TIF formation over baseline levels was not observed in MEFs expressing triple or quadruple mTIN2 mutants (Figure 6B; Supplementary information, Figure S4B). Consistent with these results, expression of single, but not triple or quadruple human TIN2 mutants in TIN2-depleted U2OS cells resulted in robust TIF formation (Supplementary information, Figure S4C and S4D). These data collectively suggest that the interactions of TIN2 with TRF1, TRF2, and TPP1 are all required to repress telomere damage signaling.

To determine the types of telomere fusions observed in TIN2-deficient cells reconstituted with TIN2 mutants, we used chromosome orientation (CO)-FISH, a cytogenetics technique which can differentiate between the telomere fusions generated by leading- and lagging-strand DNA synthesis (Supplementary information, Figure S5A). Depletion of TIN2 with shRNA increased the number of chromosome and chromatid fusions, while reconstitution with WT TIN2 repressed this fusion phenotype (Figure 6C and 6D). TIN2-depleted MEFs reconstituted with $\mathrm{mTIN} 2^{\mathrm{S} 60 \mathrm{R}}, \mathrm{mTIN}^{\mathrm{A} 103 \mathrm{R}}$, or $\mathrm{mTIN} 2^{\mathrm{L} 247 \mathrm{E}}$ all displayed robust end-to-end chromosome fusions, which are $\sim 6$-fold above fusion levels observed in cells expressing wildtype TIN2 (Figure 6C and 6D; Supplementary information, Figure S5A). Examination of fusion sites revealed that all three TIN2 mutants induced a complex mixture of chromosome- and chromatid-type fusions, including G1-type end-to-end chromosome fusions with leading and lagging telomere signal characteristic of TRF2 loss (Figure 6C-6E; Supplementary information, Figure S5A and S5B) [30-32]. In addition, we observed fusion events suggestive of postreplicative G2 repair, including chromosome fusions with unequal leading and lagging telomere signal, chromatid-type fusions involving both leading- and lagging-strand telomeres, and sister telomere fusions involving both chromatid arms reminiscent of POT1-TPP1 loss (Figure 6C, 6E; Supplementary information, Figure S5A and S5B) [10-14, 33]. These results suggest that both TIN2-TRF1 and TIN2-TRF2 interactions are required to prevent the generation of both chromosome- and chromatid-type fusions. MEFs expressing mTIN $2^{\mathrm{A} 15 \mathrm{R}}$ also displayed an increase in the number of G1- or G2-type chromosome fusions over the background levels, similar to those observed in shTin2-treated MEFs (Figure 6C and 6D). This result suggests that the TIN2-TPP1 interaction is also important to repress chro- 

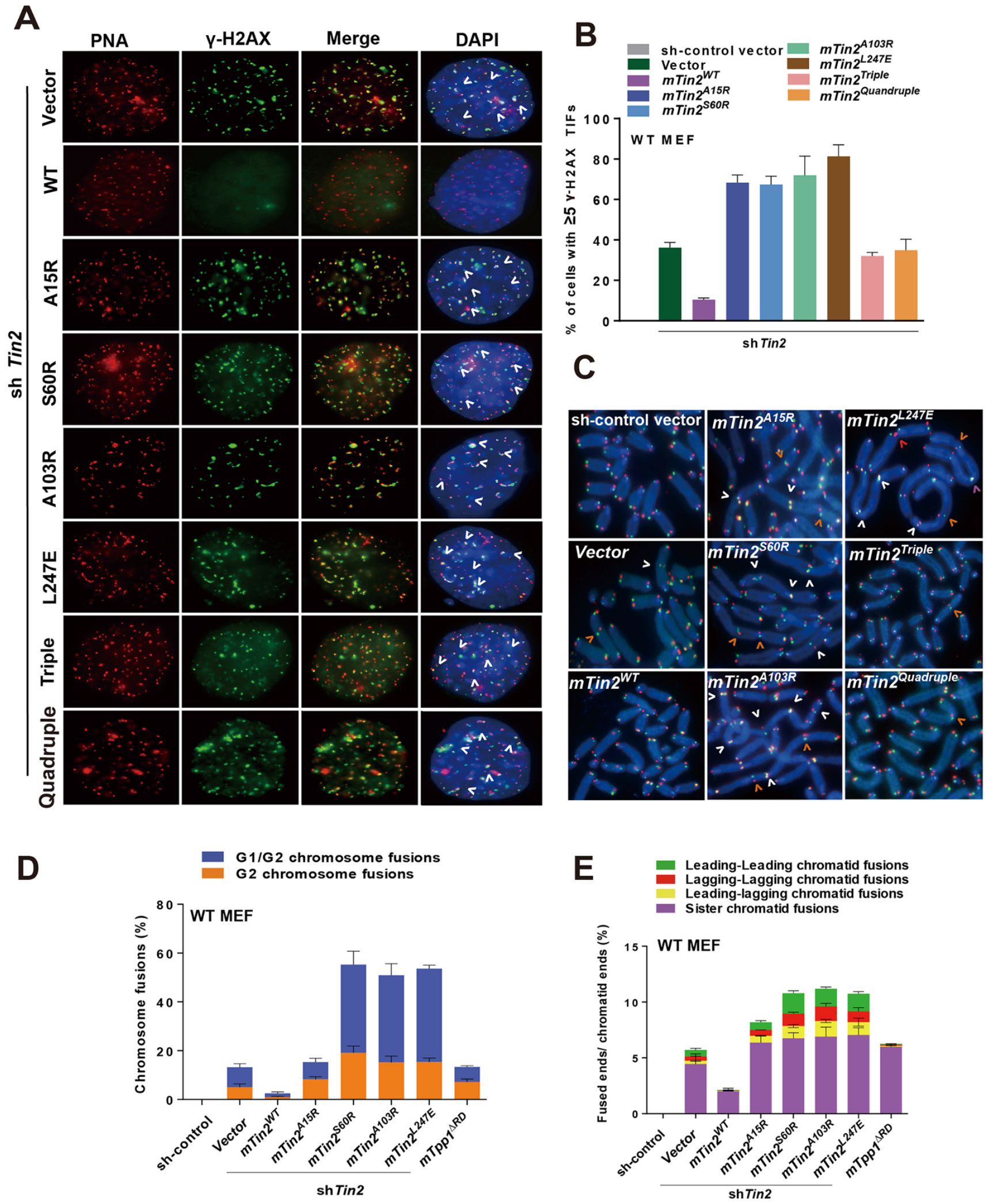
mosome fusions, although not to extent as TIN2-TRF1 and TIN2-TRF2 interactions. In addition, sister telomere fusions and chromatid fusions were abundant in MEFs expressing mTIN2 ${ }^{\mathrm{A} 15 \mathrm{R}}$, suggesting that TIN2's interaction with TPP1-POT1 is also required to repress postreplicative chromatid fusion events (Figure $6 \mathrm{C}$ and $6 \mathrm{E}$ ).

DNA double-strand breaks can be repaired either by the error-prone non-homologous end joining (alternative- or C-NHEJ)-mediated repair, or the error-free homology-directed repair (HDR) pathways. C-NHEJ is the major form of NHEJ and is mediated by the DNAPK complex (DNA-PKcs, Ku70, and Ku80) and LIG4/ XRCC4/XLF complex, while A-NHEJ does not require the above complexes [33-38]. To determine whether the TIN2 mutant-induced chromosome and chromatid fusions are a result of C- or A-NHEJ-mediated repair, we reconstituted WT or TIN2 mutants in $K u 70^{-/-}$MEFs (Supplementary information, Figure S5C and S5D). $\mathrm{Ku} 70$ is essential for C-NHEJ-mediated repair but represses A-NHEJ-mediated repair [33-38]. Thus, any chromosome fusions observed in the absence of $\mathrm{Ku} 70$ result from A-NHEJ repair. Compared to $K u 70^{+/+} \mathrm{MEFs}$, expression of $\mathrm{mTIN} 2^{\mathrm{S} 60 \mathrm{R}}, \mathrm{mTIN} 2^{\mathrm{A} 103 \mathrm{R}}$, or $\mathrm{mTIN} 2^{\mathrm{L} 247 \mathrm{E}}$ in $K u 70^{-/-}$MEFs led to a decreased number of C-NHEJ-mediated chromosome fusions, while chromatid and sister fusions increased to involve over $10 \%$ of all chromatid ends (compare Supplementary information, Figure S5ES5F with Figure 6D-6E). This result suggests that TIN2TRF1 and TIN2-TRF2 interactions are important to repress A-NHEJ-mediated chromatid and sister fusions in addition to C-NHEJ-mediated chromosome fusions. In addition, expression of $\mathrm{mTIN} 2^{\mathrm{A} 15 \mathrm{R}}$ in $\mathrm{Ku} u 0^{-/-} \mathrm{MEFs}$ resulted in increased sister telomere fusions and chromatid-type fusions compared to WT MEFs, suggesting that the TIN2-TPP1 complex is also involved in repression of A-NHEJ-mediated chromatid and sister telomere fusions (Figure 6E; Supplementary information, Figure S5F). Taken together, our data suggest that TIN2-TRF1 and TIN2-TRF2 interactions are essential to repress telomere damage signaling, preventing both C-NHEJ-mediated chromosome fusions and A-NHEJ-mediated chromatid fusions. The TIN2-TPP1 interaction is required to repress A-NHEJ-mediated chromatid, sister telomere fusions, and to a smaller extent, C-NHEJ-mediated chromosome fusions.

\section{Discussion}

Shelterin regulates telomere maintenance and inhibits DDR at telomeres [2]. In the present study, we report the crystal structure of N-terminal domain of TIN2 in complex with TIN2-binding motifs from TPP1 and TRF2, which represents the central core shelterin subcomplex. Our structural analyses finally complete the high-resolution views of all building blocks of the shelterin complex (Figure 7A and 7B).

Our current data and previous structural analyses suggest that all of the shelterin subcomplexes utilize a domain-peptide-interaction mechanism (Figure 7A). The TRF1/TRF2 TRFH domain recognizes a conserved TBM [4], while the RCT domain of RAP1 interacts with a helical peptide of TRF2 adjacent to TRFH domain [39]. The POT1 C-terminal region including an $\mathrm{OB}$ fold and a Holliday junction-like domain binds with a long fragment of TPP1 [40, 41]. Here, we show that TIN2 TRFH domain binds to short motifs from TPP1 and TRF2 to form the TIN2-TPP1-TRF2 subcomplex. This domain-peptide-interaction mechanism is also employed by telomeric protein complexes from other species, including the fission yeast $S p R a p 1_{R C T}-T a z 1_{R B M}$ complex [39], the budding yeast $S c$ Rap $1_{\text {RCT }}$-Sir $3_{\mathrm{RBM}}$ [39], and $S c$ Rap $1_{\mathrm{RCT}}-$ Rifl $_{\mathrm{RBM}}$ complexes [42]. This conserved domain-peptide-interaction mechanism provides the possibility for dynamic regulation of complex formation or dissociation. Any modification on a single residue could abolish the binding affinity between a domain and a peptide due to the relatively small contacting interface. This notion is supported by our recent work showing that the

Figure 6 TIN2 protects telomeres from DNA damage signaling and chromosome fusions. (A) Colocalization of $\gamma$-H2AX with telomeres in reconstituted WT or indicated mTIN2 mutants. Telomeres were visualized by PNA-FISH (red), anti- $\gamma$-H2AX antibody to visualize DNA damage (green), and DAPI to visualize nuclei (blue). (B) Quantification of the percentage of cells containing $\geq 5$ positive $\gamma$-H2AX TIFs in (A). Data represent the mean of two independent experiments \pm SEM; more than 125 nuclei were analyzed per experiment. (C) MEFs expressing either WT or MTIN2 mutants were infected with either control vector or shTin2 for $120 \mathrm{~h}$, after which metaphases were prepared and telomere fusions were visualized by CO-FISH.

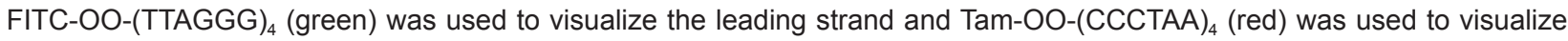
the lagging strand. DAPI (blue) visualized chromosomes. Arrowheads point to fused chromosomes: chromosome fusions (white arrows), and an unequal mixture of leading and lagging G2 chromosome fusions (orange arrows). (D) Quantification of chromosome-type fusions in (C). (E) Quantification of chromatid-type and sister fusions in (C). $\mathrm{mTPP} 1^{\triangle \mathrm{RD}}$ is a dominant-negative mutant of mTPP1 in which POT1-binding recruitment domain (RD) is deleted. Expression of $m$ TPP $1^{\triangle R D}$ in MEFs will remove endogenous TPP1-POT1a/b complex from telomeres. Data represent the average of two independent experiments as mean \pm SEM from more than 70 metaphases examined. 


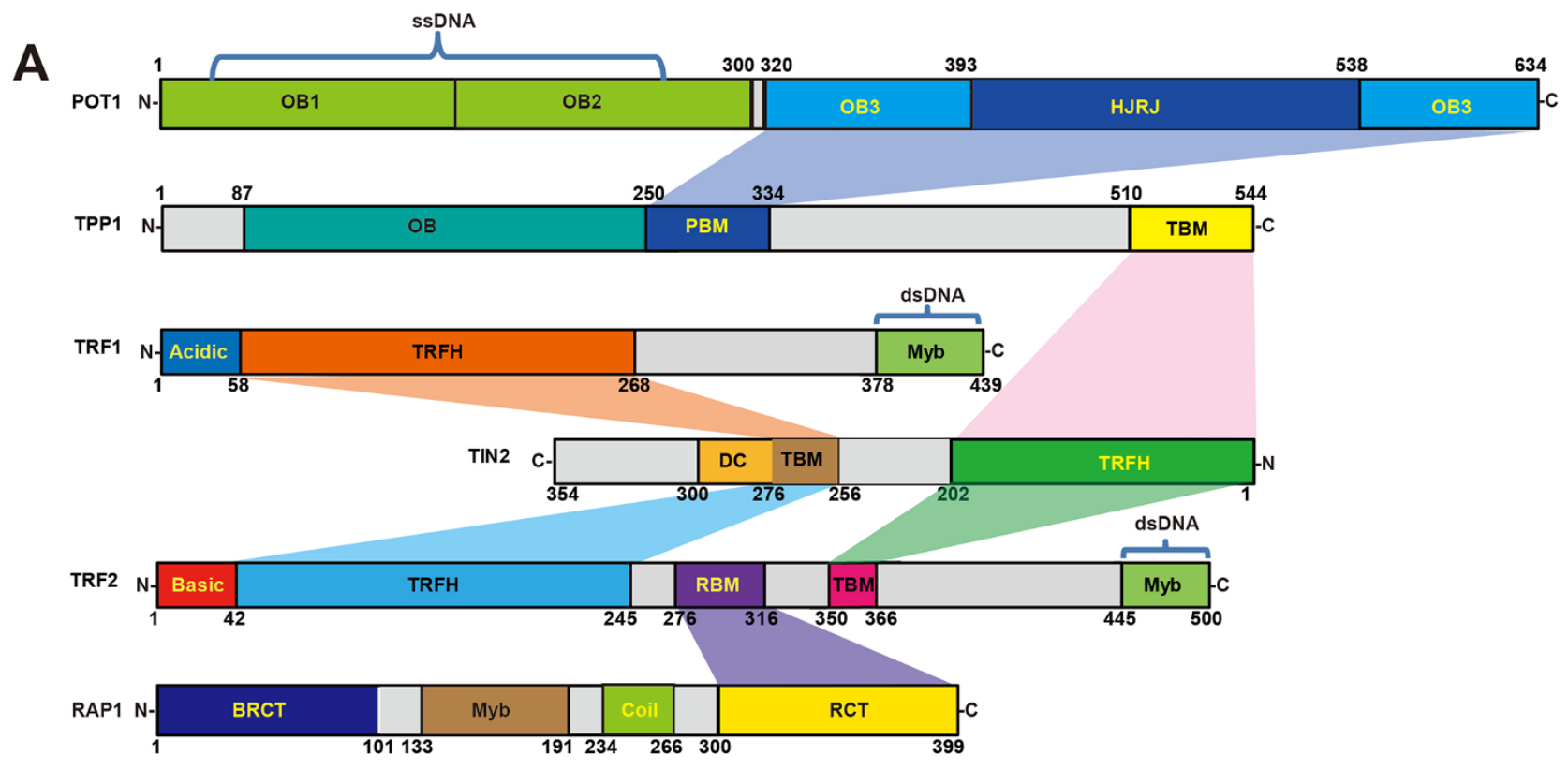

B

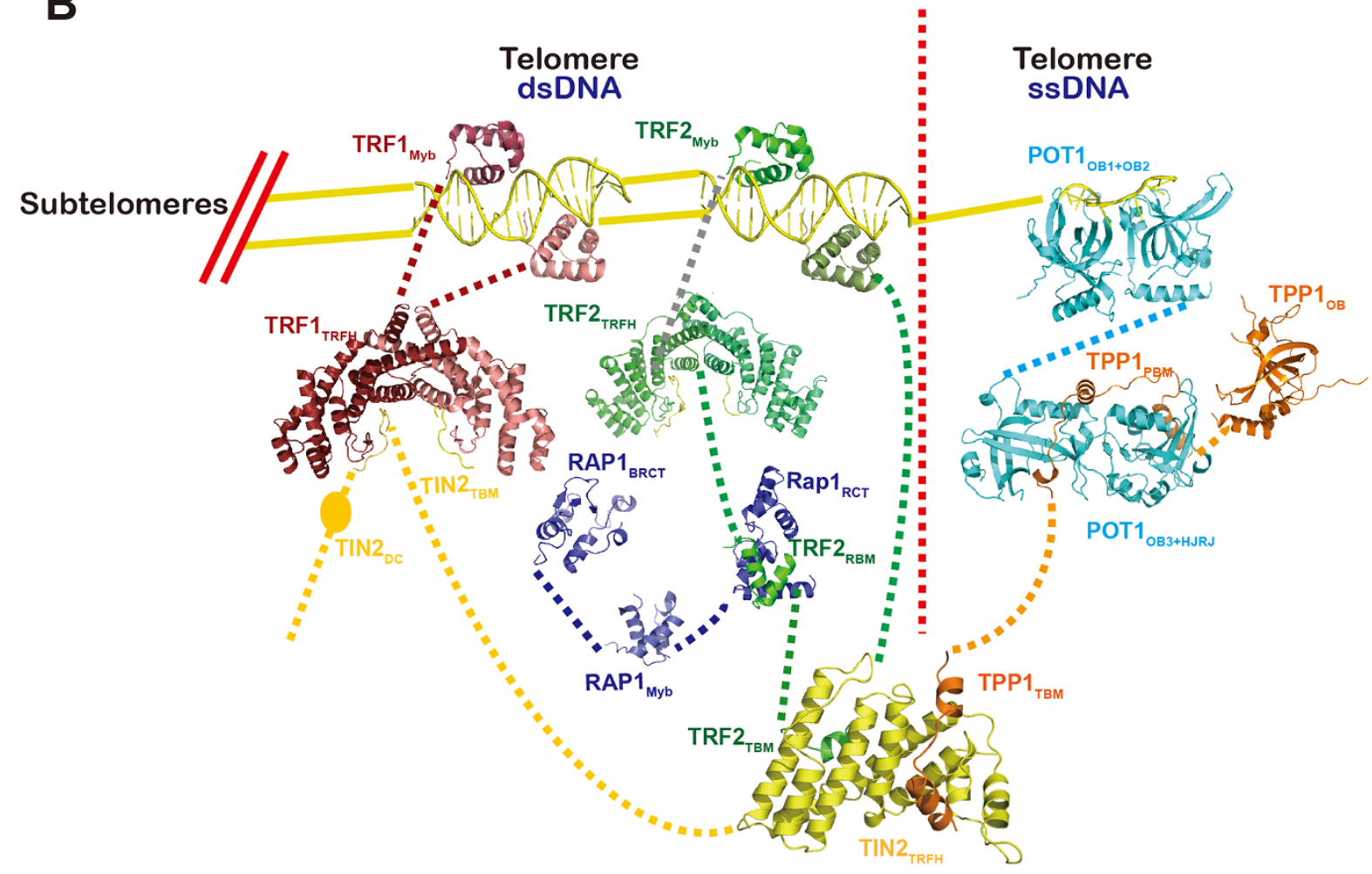

Figure 7 The structure of human shelterin complex. (A) Domain organizations of the shelterin components. (B) The structural model of human shelterin complex based on current available structures, including TRF1 $1_{\text {Myb }}$-dsDNA (PDB: 1W0T), TRF$2_{\mathrm{Myb}}$-dsDNA (PDB: 1WOU), POT1 $1_{\mathrm{OB} 1+\mathrm{OB} 2}$-SSDNA (PDB: $\left.1 \mathrm{XJV}\right), \mathrm{TRF}_{\mathrm{TRFH}}$ in complex with TIN2 ${ }_{\mathrm{TBM}}(\mathrm{PDB}: 3 \mathrm{BQO}), \mathrm{TRF}_{\mathrm{TRFH}}$ in

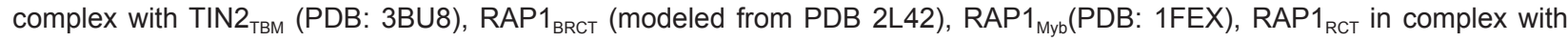

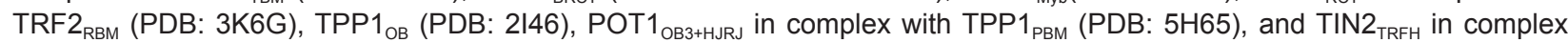

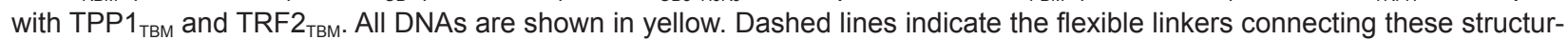
al modules. For clear illustration purpose, only one RAP1 and TIN2 are presented, and only one TRF2 $2_{\text {TBM }}$ and TRF2 $2_{\text {RBM }}$ from a TRF2 monomer are shown. 
interaction between TRF 2 and NBS1 is modulated by NBS1's cell-cycle-dependent phosphorylation status [6]. The assembly of other shelterin subcomplexes, which utilizes this domain-peptide mechanism, may also be susceptible to regulation by similar cell-cycle-dependent or DDR-dependent phosphorylation events. The structure of TIN2-TRF2-TPP1 shown here implies a potential regulatory mechanism for the stability of TIN2-TRF2 complex. Indeed, TRF $2^{\mathrm{T} 358}$ in the TIN2-binding motif is known to be phosphorylated by Aurora C [43]. Our crystal structure reveals that $\mathrm{TRF} 2^{\mathrm{T} 358}$ sits in an acidic pocket of TIN2 (Figure 4A), and thus a negatively charged phosphate group deposited on T358 would weaken or disrupt the interaction between TIN2 and TRF2. Additionally, we found that TRF2 contains a highly conserved PIKK phosphorylation site at S368, immediately following the TIN2-binding motif of TRF2 (residues 350-366). Since the C-terminus of TRF2 $2_{\text {TвM }}$ is close to a basic surface of TIN2, phosphorylation of S368 is expected to enhance the TIN2-TRF2 binding. Whether the assembly of the TIN2-TRF2-TPP1 complex is modulated by such phosphorylation events in vivo is currently being investigated.

The most striking finding in this study is that TIN $2_{\mathrm{TRFH}}$ adopts a TRFH-like fold. TRFH domain was first identified in human TRF1 and TRF2 [44, 45]. Despite the high degree of structural similarity, there are some differences between TIN $2_{\text {TRFH }}$ and the TRFH domains of TRF1 and TRF2. The TIN $2_{\text {TRFH }}$ does not have the equivalent $\alpha 1$, $\alpha 2$, and $\alpha 10$ helices in TRF1/2 $2_{\text {TRFH }}$ (Figure 2A), which are important for TRF $1 / 2_{\mathrm{TRFH}^{-}}$-mediated dimerization of TRF $1 / 2$ proteins [44]. Instead, the C-terminal $\alpha 8$ and $\alpha 9$ helices of TIN $2_{\text {TRFH }}$ occupy the equivalent positions of helices $\alpha 1$ and $\alpha 2$ in the TRFH domains of TRF1 and TRF2, blocking the dimerization interface found in TRF$1_{\text {TRFH }}$ and TRF $2_{\text {TRFH }}$ (Figure 2A). In agreement with this observation, TIN $2_{\mathrm{TRFH}}$ exists as a monomer in solution, as revealed by gel-filtration chromatography analysis and multiangle light scattering (Supplementary information, Figures S1C and S6A). Recently, TRFH domain of TRF2 has been reported to bind and wrap DNA to regulate the topology of telomeres [46]. The DNA-binding activity of TRF $2_{\text {TRFH }}$ relies on seven lysine and two arginine residues. Structure-based alignment showed that TIN2 only has two lysine residues at the equivalent positions (Supplementary information, Figure S6B), so it is predicted that TIN2 $2_{\text {TRFH }}$ may not have the DNA-binding capacity. Electrophoresis mobility shift assays confirmed that TIN $2_{\text {TRFH }}$ cannot bind to dsDNA as TRF $2_{\text {TRFH }}$ does (Supplementary information, Figure S6C). These analyses indicate that TRFH domain might be an ancient domain in telomere-binding proteins and has gained significant structural and functional plasticity during evolution.
The TIN2-TPP1-TRF2 structural model rationalizes previously reported mutagenesis data showing that TIN2 binding to TPP1 was abolished in TIN2 mutants such as $\Delta \mathrm{N} 18, \mathrm{~F} 37 \mathrm{D} / \mathrm{L} 38 \mathrm{E}$, and L48E [47], as these key residues on the $\alpha 1$ and $\alpha 2$ helices of TIN2 are required to mediate hydrophobic contacts with $\mathrm{TPP} 1_{\mathrm{TBM}}$ (Figure 3A and 3B). Frescas et al. [18] has generated a TIN2 mutant (TIN $2^{\Delta 132-188}$ ) to specifically remove TIN2-TPP1 interaction while not affecting TIN2-TRF2 and TIN2TRF1 interactions. TIN $2^{132-188}$ contains helices $\alpha 6, \alpha 7$, $\alpha 8$, and the starting turn of $\alpha 9$. The helices $\alpha 7, \alpha 8$, and the connecting loop $\mathrm{L}_{78}$ of TIN2 make extensive contacts with ТPР $1_{\text {твм }}$ (Figure $3 \mathrm{~A}, 3 \mathrm{~B}$; Supplementary information, Figure S7A), so deletion of TIN $2^{132-188}$ will disrupt the interaction with TPP1. However, $\alpha 6$ and $\alpha 7$ are also involved in TRF2 interaction, so deletion of residues 132-188 might impair the interaction with TRF2 as well (Supplementary information, Figure S7A). More importantly, helices $\alpha 6-\alpha 8$ are the integral part of a compact helical bundle (Figure 1B), making extensive hydrophobic contacts with the rest of TIN $2_{\mathrm{TRFH}}$ (Supplementary information, Figure S7B). In particular, $\alpha 7$ is dominantly composed of hydrophobic residues and is surrounded by a hydrophobic groove formed by $\alpha 1, \alpha 2$, and $\alpha 3$ of TIN2 (Supplementary information, Figure S7C). Thus, deletion of TIN $2^{132-188}$ is expected to disrupt the overall fold of TIN $2_{\text {TRFH }}$. This prediction is confirmed by the fact that the deletion of helices $\alpha 8-\alpha 9$ (TIN $2^{2-160}$ construct) cannot yield a soluble protein (Supplementary information, Figure S7D). Thus, the TIN2 mutant that lacks residues 132-188 is not a good candidate to mimic the TPP1-binding-deficient TIN2 allele. For this reason, in the present study, we designed point mutations, instead of fragment deletions, to specifically disrupt the TIN2 interactions with binding partners and to dissect the functions of TIN2 in the cells.

Our structure-based mutational analyses shed light on how specific shelterin subcomplexes repress distinct DNA damage signaling and repair mechanisms at telomeres. We found that the TIN2 $2^{\mathrm{A} 15 \mathrm{R}}$ mutant, which is unable to interact with TPP1 but localizes to telomeres through interactions with TRF2 and TRF1, induced a robust DDR, increased chromatid and sister telomere fusions, and slightly increased in chromosome fusions (Figure 6D and 6E). It is unclear whether these chromosome fusions arose from G1 fusions or postreplicative G2 fusions. End-to-end chromosome fusion can also arise from duplication of the chromatid-type fusions formed in the preceding G2 [48]. These phenotypes bear some resemblance to those observed when the POT1-TPP1 heterodimer is depleted $[10-14,33]$. It suggests that the major function of TIN2-TPP1 interaction is to load POT1- 
TPP1 heterodimer to telomeres to protect telomeres from engaging in ATR-dependent DNA damage signaling and A-NHEJ-mediated repair at telomeres, consistent with previous TIN2 knockout studies [22]. On the contrary, mTIN $2^{\mathrm{S} 60 \mathrm{R}}, \mathrm{mTIN}^{\mathrm{A} 103 \mathrm{R}}$, and mTIN2 $2^{\mathrm{L} 247 \mathrm{E}}$ mutants induced complicated phenotypes reminiscent of both TRF2 loss and POT1-TPP1 loss, including both chromosome and chromatid fusions [10-12, 30-33, 38]. This result suggests that both TIN2-TRF1 and TIN2-TRF2 interactions are required for shelterin complex stability. This is consistent with the previous report that TIN2 depletion or overexpression of TIN2-15, a dominant negative mutant which cannot interact with TRF1, destabilized both TRF1 and TRF2 $[17,20]$. Taken together, our mutagenesis data provided a good starting point to fully understand the functional significance of TIN2-mediated interactions in telomere protection.

In the past few decades, structural studies of shelterin components have been greatly advanced to facilitate better understanding of the molecular mechanisms of telomere maintenance. Although the flexible nature of shelterin complex impedes structural determination of the intact complex, now, all the structural domains and subcomplexes have been structurally characterized (Figure 7B). The next challenge will be to decipher the structure and dynamic regulation of the full shelterin complex to reveal how these subcomplexes, or functional units, coordinate for telomere replication and protection.

\section{Materials and Methods}

\section{Protein expression and purification}

Human TIN2 ${ }_{\text {TRFH }}$ (residues 2-202) (GenBank: AAF18439.1) and TPP $1_{\text {твм }}$ (residues 510-544) (GenBank: AAS80318.1) fragments were cloned into modified pET-Duet vector, TIN $2_{\text {TRFH }}$ into promoter I, and TPP $1^{\text {TBM }}$ into promoter II. A $6 \mathrm{x}$ His tag and a $3 \mathrm{C}$ protease site (LEVLFQGP) were fused at its $\mathrm{N}$-terminus of TIN $2_{\text {TRFH }}$. The TIN $2_{\text {TRFH }}-$ TPP $1_{\text {TBM }}$ complex was coexpressed in Escherichia coli BL21 (DE3). After induction for $16 \mathrm{~h}$ with 0.4 $\mathrm{mM}$ IPTG at $25^{\circ} \mathrm{C}$, the cells were harvested by centrifugation, and the pellets were resuspended in lysis buffer $(50 \mathrm{mM}$ Tris- $\mathrm{HCl}, \mathrm{pH}$ 8.0, $400 \mathrm{mM} \mathrm{NaCl}, 3 \mathrm{mM}$ imidazole, $10 \%$ glycerol, $1 \mathrm{mM}$ PMSF, $2 \mathrm{mM} 2$-mercaptoethanol, and homemade protease inhibitor cocktail). Cells were lysed by sonication followed by centrifugation to remove the cell debris. The supernatant was mixed with Ni-NTA agarose beads (Qiagen) and rotated for $2 \mathrm{~h}$ at $4{ }^{\circ} \mathrm{C}$ before elution with $300 \mathrm{mM}$ imidazole. $3 \mathrm{C}$ protease was added at a molar ratio of 1:100 to remove the $6 \mathrm{x}$ His tag in the N-terminus of TIN $2_{2-202}$. After $3 \mathrm{C}$ digestion, the TIN2 $2_{2-202}-\mathrm{TPP} 1_{\text {твм }}$ complex was further purified by gel-filtration chromatography on Hiload Superdex 200 column (GE Healthcare) equilibrated with buffer (25 mM Tris$\mathrm{HCl}, \mathrm{pH} 8.0,150 \mathrm{mM} \mathrm{NaCl})$. The purified TIN $2_{\mathrm{TRFH}}-\mathrm{TPP} 1_{\mathrm{TBM}}$ complex was concentrated to $25 \mathrm{mg} / \mathrm{mL}$ and stored at $-80{ }^{\circ} \mathrm{C}$.

Human TRF $2_{\text {твм }}$ (residues 350-366) (GenBank: AAB81135.1) was expressed in E. coli BL21 (DE3) using a modified pET28b vec- tor with a SUMO protein fused at the N-terminus after the $6 \mathrm{x}$ His tag. After induction for $5 \mathrm{~h}$ with $0.1 \mathrm{mM} \mathrm{IPTG}$ at $37^{\circ} \mathrm{C}$, the cells were harvested by centrifugation, and the pellets were resuspended in lysis buffer (50 mM Tris- $\mathrm{HCl}, \mathrm{pH} 8.0,400 \mathrm{mM} \mathrm{NaCl}, 3 \mathrm{mM}$ imidazole, 10\% glycerol, $1 \mathrm{mM}$ PMSF, $2 \mathrm{mM}$ 2-mercaptoethanol, and homemade protease inhibitor cocktail). The cells were then lysed by sonication, followed by centrifugation to remove cell debris. The supernatant was mixed with Ni-NTA agarose beads (Qiagen) and rotated for $2 \mathrm{~h}$ at $4{ }^{\circ} \mathrm{C}$ before elution with $250 \mathrm{mM}$ imidazole. Ulp1 protease was added at a molar ratio of 1:200 to remove the His-SUMO tag. After Ulp1 digestion, the TRF2 $2^{\mathrm{TBM}}$ peptide was further purified by gel-filtration chromatography on Hiload Superdex75 column (GE Healthcare) equilibrated with $150 \mathrm{mM} \mathrm{NH}_{4} \mathrm{HCO}_{3}$ solution. The fractions containing the peptides were collected and lyophilized.

Crystallization, data collection, and structure determination The TIN $2_{\mathrm{TRFH}^{-}}-\mathrm{TPP} 1_{\mathrm{TBM}}$ complex and TRF $2_{\text {TBM }}$ peptide were mixed at a molar ratio of 1:2 and the mixtures were used for crystallization. Crystal screening was performed with Hampton-screening kit by sitting-drop-vapor diffusion at $20{ }^{\circ} \mathrm{C}$. The precipitant/well solutions are solution A for native sample crystals and solution $\mathrm{B}$ for selenomethionine (Se-Met) sample crystals (solution A: $0.2 \mathrm{M}$ calcium acetate hydrate, $20 \%$ polyethylene glycol 3350 , and $2 \mathrm{mM}$ DTT; solution B: 0.1 M Tris-HCl, pH 8.5, 0.2 M magnesium chloride, $25 \%$ polyethylene glycol 3350 , and $2 \mathrm{mM} \mathrm{DTT}$ ). All crystals were gradually transferred into harvesting solutions (precipitant solution and $25 \%$ glycerol) before being flash-frozen in liquid nitrogen. SeMet-SAD diffraction data were screened and collected at the beamlines BL18U and BL19U1 of the Shanghai Synchrotron Radiation Facility with a wavelength of $0.979 \AA$ at $100 \mathrm{~K}$, and processed using HKL2000 [49]. Six selenium sites were located and refined, and the single-wavelength anomalous diffraction data phases were calculated with SHARP [50]. The structure refinement was done in PHENIX package [51] with manual rebuilding in COOT [52].

\section{Yeast two-hybrid screening}

Yeast cells growth and manipulation were done according to standard procedures. The yeast strain L40 (MATa his $3 \Delta 200$ trp1-901 leu2-3112 ade2 LYS::(4lexAop-HIS3) URA3::(81exAop-LacZ)GAL4) was used in this study. The yeast two-hybrid assays were performed with two plasmids pBTM116 (binding domain) and pACT2 (activation domain). The colonies containing both plasmids were selected on -Leu -Trp plates. The $\beta$-galactosidase activities were measured by liquid assay according to the standard manual.

\section{Microscale thermophoresis assay}

MST is a novel immobilization-free technique for the analysis of molecular interactions. MST measurements were performed with a NanoTemper Monolith NT.115 instrument (NanoTemper Technologies). In brief, in this study, $50 \mathrm{nM}$ NT-650 (NanoTemper Technologies)-labeled TRF2 full-length proteins $(10 \mu \mathrm{L})$ were first incubated for $10 \mathrm{~min}$ on ice with different concentrations of the TIN2FL, TIN2N, and TIN2C proteins $(10 \mu \mathrm{L})$, respectively, in $1 \times$ PBS buffer ( $\mathrm{NaCl} 137 \mathrm{mM}, \mathrm{KCl} 2.7 \mathrm{mM}, \mathrm{Na}_{2} \mathrm{HPO}_{4} 10 \mathrm{mM}$, and $\mathrm{KH}_{2} \mathrm{PO}_{4} 2 \mathrm{mM}$, pH 7.4). Then, $5 \mu \mathrm{L}$ of the samples was loaded into standard treated capillaries, and MST measurements were col- 
lected at $25{ }^{\circ} \mathrm{C}$ at $30 \%$ infrared-laser power and $60 \%$ light-emitting-diode power. The laser-on and laser-off intervals were 30 and $5 \mathrm{~s}$, respectively. NanoTemper Analysis 1.2.20 software was used to fit the data and to determine the apparent $K_{\mathrm{d}}$ values. All measurements were collected at least three times.

\section{Fluorescence polarization}

Different ligand proteins were diluted to a series of concentrations from $6 \mathrm{nM}$ to $200 \mu \mathrm{M}$ in $20 \mathrm{mM}$ HEPES, pH 7.4, $150 \mathrm{mM}$ $\mathrm{NaCl}$, and $10 \%$ glycerol. The FAM-labeled molecules were used at a final concentration of $100 \mathrm{nM}$. The final volume was brought up to $100 \mu \mathrm{L}$ with dilution buffer (20 mM HEPES, pH 7.8, $150 \mathrm{mM} \mathrm{NaCl}$, and $10 \%$ glycerol) and incubated in the dark for $30 \mathrm{~min}$. The fluorescence polarization values were measured using Synergy Neo Multi-Mode Reader (Bio-Tek) at $25{ }^{\circ} \mathrm{C}$. Excitation wavelength was $485 \mathrm{~nm}$ and emission was detected at $528 \mathrm{~nm}$. Fluorescence was quantitated with GEN 5 software and data were analyzed with Prism 6.

\section{Electrophoretic mobility shift assay}

The sequence of DNA template used for the assay is 5'-CTGGATCCNNNNNNNNNNNNNNNNNNNNNGTCGACAAGCTTCTCGAGAC-3'. The dsDNA was obtained by annealing this $(\mathrm{N})_{21}$-containing template with a FAM-labeled oligo (5'-GTCTCGAGAAGCTTGTCGAC-3'), followed by filling of $5^{\prime}$ overhangs to form blunt ends using Klenow enzyme. Various concentrations of proteins in binding buffer $(25 \mathrm{mM}$ Tris- $\mathrm{HCl}, \mathrm{pH} 8.0,150 \mathrm{mM} \mathrm{NaCl}$, 2 mM DTT, and 10\% glycerol) were mixed with 50 nM FAM-labeled dsDNA in a total volume of $15 \mu \mathrm{L}$. The reaction mixtures were incubated at room temperature for $30 \mathrm{~min}$ before being loaded onto a $4 \%$ $20 \%$ nondenaturing polyacrylamide gel. The gels were then dried and visualized on Bio-Rad PharosFX Plus.

Size-exclusion chromatography coupled with multiangle light scattering

About $20-\mu \mathrm{L}$ protein samples $(2 \mathrm{mg} / \mathrm{mL}$ in $50 \mathrm{mM}$ Tris- $\mathrm{HCl}$, $\mathrm{pH} 8.0,150 \mathrm{mM} \mathrm{NaCl}$ ) were analyzed with static light scattering by injecting them into an Agilent 1260 HPLC system with a SEC column (Wyatt Technology, WTC-030S5). The chromatography system was coupled with an 18-angle light-scattering detector (DAWN HELEOS II, Wyatt Technology) and differential refractive index detector (Optilab T-rEx, Wyatt Technology). Masses (molecular weights) were calculated with ASTRA (Wyatt Technology). Bovine serum albumin (Sigma) was used as the calibration standard.

\section{Co-immunoprecipitation}

293 T cells were transiently transfected by lipofectamine 3000 using $4 \mu \mathrm{g}$ of total plasmid DNA per well in six-well dishes. Cells were lysed in IP buffer (20 mM Tris-HCl, pH 7.4, $150 \mathrm{mM} \mathrm{NaCl}$, $0.05 \%$ Triton X-100, 1 mM EDTA and EGTA, 10\% glycerol, $1 \mathrm{mM}$ DTT, and $1 \mathrm{mM}$ phenylmethylsulfonyl fluoride), and supernatants were used for immunoprecipitation with Flag-M2-beads (Sigma). Beads were washed four times and then proteins were eluted and analyzed by SDS-PAGE.

\section{Antibodies and western analysis}

The antibodies used for western blot analysis are as follows: rabbit anti-phospho-CHK1 (Cell Signaling Technology, 2348), mouse anti-phospho-CHK2 (BD Biosciences, 611570), mouse anti- $\gamma$-H2AX (Millipore, 05-636), mouse anti-TRF2 (Millipore, 05521), rabbit anti-53BP1 (Santa Cruz, sc-22760), mouse anti-Flag (Sigma-Aldrich, F3165), mouse anti- $\gamma$-tubulin (clone GTU-488, Sigma-Aldrich, T6557), mouse anti-TRF1 (Abcam, ab10579). Rabbit anti-mTRF2 antibody was a gift from J Karlseder. Rabbit anti-mTPP1 antibody was generated using the peptide sequence CSQLLDEVREDQDHR. For immunoblotting, trypsinized cells were lysed in urea lysis buffer $(8 \mathrm{M}$ urea, $50 \mathrm{mM}$ Tris- $\mathrm{HCl}, \mathrm{pH}$ 7.4, and $150 \mathrm{mM} \beta$-mercaptoethanol). The lysate was resolved on SDS-PAGE gel and separated proteins were then blotted on a PVDF membrane (Amersham), blocked with blocking solution (5\% nonfat dry milk in PBS $/ 0.1 \%$ Tween 20$)$ for $1 \mathrm{~h}$, and incubated with the appropriate primary antibody in blocking solution for $2 \mathrm{~h}$ at room temperature or overnight at $4{ }^{\circ} \mathrm{C}$. The membranes were washed for $3 \times 5$ min with PBS/ $0.1 \%$ Tween 20 and incubated with the appropriate secondary antibody in blocking solution for $1 \mathrm{~h}$ at room temperature. Chemiluminescence detection was performed using an ECL Western Blotting Detection kit from GE Healthcare.

\section{Expression vectors and shRNAs}

Human and mouse WT TIN2 and mutants were cloned into pQCXIP puro retroviral expression vectors. All the constructs were confirmed by sequencing. Point mutations and shRNA-resistant sequence were introduced using side-directed mutagenesis (Stratagene). Lenti shRNA against mTIN2 (TRCN0000305996, TRCN0000305925) was purchased from Sigma. shRNA against hTIN2 was from [47].

\section{Retroviral infections}

For retroviral infection, DNA constructs were transfected into 293 T cells using Fugene 6 and packaged into viral particles. Viral supernatant was collected $48-72 \mathrm{~h}$ after transfection, filtered, and directly used to infect immortalized MEFs.

\section{Immunofluorescence and fluorescent in situ hybridization}

Cells grown on coverslips were fixed for $10 \mathrm{~min}$ in $2 \%(\mathrm{w} /$ v) sucrose and $2 \%(\mathrm{v} / \mathrm{v})$ paraformaldehyde at room temperature followed by PBS washes. Coverslips were blocked in $0.2 \%$ (w/ v) fish gelatin and $0.5 \%(\mathrm{w} / \mathrm{v}) \mathrm{BSA}$ in PBS. Cells were incubated with primary antibodies and after PBS washes, cells were incubated with appropriate Alexa Fluor secondary antibodies followed by washes in PBS $+0.1 \%$ Triton X-100. IF-FISH was carried out using a 5'-Tam-OO-(CCCTAA) $)_{4}$-3' PNA telomere probe (PANAgene). DNA was stained with DAPI, and digital images were captured using Metamorph (Molecular Devices) with a Nikon Eclipse 800 microscope and an Andore CCD camera. Cells with $\geq 5 \gamma$-H2AX or 53BP1-positive signals colocalizing with telomere signals are considered as TIF-positive cells [53, 54].

Chromosome analysis by telomere PNA-FISH and CO-FISH

Cells were treated with $0.5 \mu \mathrm{g} / \mathrm{mL}$ of Colcemid before harvest. Chromosomes were fixed and telomere PNA-FISH was performed with a 5'-Tam-OO-(CCCTAA) $)_{4}-3^{\prime}$ probe (PANAgene) as described $[12,54]$. CO-FISH was used to detect both newly synthesized leading- and lagging-strand telomeric DNAs [6, 33]. For CO-FISH, metaphase spreads were incubated sequentially with 5'-Tam-OO-(CCCTAA) $)_{4}-3^{\prime}$ and 5'-FITC-CO-(TTAGGG) $)_{4}-3^{\prime}$ 
probes. Images were captured as above. The percent of chromosome fusions observed is defined as the total number of fused chromosomes in 30-50 metaphase spreads divided by the total number of chromosomes examined $\times 100 \%$. The frequency of chromatid-type and sister fusions was quantified as the number of fused chromatid (sister) ends/total number of chromatid ends $\times$ $100 \%$.

\section{Accession codes}

Coordinate and structure factors have been deposited in the Protein Data Bank under accession codes $5 \mathrm{XYF}\left(\mathrm{TIN} 2_{\mathrm{TRFH}^{-}}\right.$ $\left.\mathrm{TPP} 1_{\text {твм }}-\mathrm{TRF} 2_{\text {Твм }}\right)$.

\section{Acknowledgments}

We thank staffs from BL18U1 and BL19U1 beamlines at NCPSS and Shanghai Synchrotron Radiation Facility (SSRF) for their help with crystal data collection. We are extremely grateful to National Center for Protein Sciences Shanghai (Protein Expression and Purification system, NMR system) for their instrument support and technical assistance. This work was supported by grants from the Strategic Priority Research Program of the Chinese Academy of Sciences (XDB08010201) to ML and YC, the Ministry of Science and Technology of China (2013CB910401 to YC,2013CB910402 to ML), the National Natural Science Foundation of China (31470737 and 31670748 to YC, 31330040 and 31525007 to ML), the Basic Research Project of Shanghai Science and Technology Commission (14JC1407200 to YC), and NCI (RO1 CA129037, RO1CA202816, R21CA200506, and R21CA182280) to $\mathrm{SC}$.

\section{Author Contributions}

$\mathrm{YC}, \mathrm{SC}$, and ML conceived this study. CHu, JX, and YX purified the proteins. $\mathrm{CHu}$ and $\mathrm{YC}$ performed crystallization and $\mathrm{X}$-ray crystallography analyses. $\mathrm{CHu}$ performed MST analyses. $\mathrm{CHu}$, CHuang, and JL performed yeast two-hybrid assays. CHuang and JL carried out the Co-IP analyses. RR and BC carried out the IF, FISH, and CO-FISH experiments and analyzed the data. $\mathrm{CHu}, \mathrm{RR}$, CHuang, BC, and YC prepared the figures. YC, RR, SC, and $\mathrm{ML}$ wrote the manuscript.

\section{Competing Financial Interests}

The authors declare no competing financial interests.

\section{References}

1 Palm W, de Lange T. How shelterin protects mammalian telomeres. Annu Rev Genet 2008; 42:301-334.

2 de Lange T. Shelterin: the protein complex that shapes and safeguards human telomeres. Genes Dev 2005; 19:2100-2110.

3 de Lange T. How telomeres solve the end-protection problem. Science 2009; 326:948-952.

4 Chen Y, Yang Y, van Overbeek M, et al. A shared docking motif in TRF1 and TRF2 used for differential recruitment of telomeric proteins. Science 2008; 319:1092-1096.

5 Kim $\mathrm{H}$, Lee $\mathrm{OH}$, Xin $\mathrm{H}$, et al. TRF2 functions as a protein hub and regulates telomere maintenance by recognizing specific peptide motifs. Nat Struct Mol Biol 2009; 16:372-379.
6 Rai R, Hu C, Broton C, Chen Y, Lei M, Chang S. NBS1 phosphorylation status dictates repair choice of dysfunctional telomeres. Mol Cell 2017; 65:801-817.e4.

7 Wang F, Podell ER, Zaug AJ, et al. The POT1-TPP1 telomere complex is a telomerase processivity factor. Nature 2007; 445:506-510.

8 Xin H, Liu D, Wan M, et al. TPP1 is a homologue of ciliate TEBP-beta and interacts with POT1 to recruit telomerase. $\mathrm{Na}$ ture 2007; 445:559-562.

9 Nandakumar J, Bell CF, Weidenfeld I, Zaug AJ, Leinwand LA, Cech TR. The TEL patch of telomere protein TPP1 mediates telomerase recruitment and processivity. Nature 2012; 492:285-289.

10 Denchi EL, de Lange T. Protection of telomeres through independent control of ATM and ATR by TRF2 and POT1. Nature 2007; 448:1068-1071.

11 Guo X, Deng Y, Lin Y, et al. Dysfunctional telomeres activate an ATM-ATR-dependent DNA damage response to suppress tumorigenesis. EMBO J 2007; 26:4709-4719.

12 Wu L, Multani AS, He H, et al. Pot1 deficiency initiates DNA damage checkpoint activation and aberrant homologous recombination at telomeres. Cell 2006; 126:49-62.

13 He H, Multani AS, Cosme-Blanco W, et al. POT1b protects telomeres from end-to-end chromosomal fusions and aberrant homologous recombination. EMBO J 2006; 25:5180-5190.

14 Hockemeyer D, Daniels JP, Takai H, de Lange T. Recent expansion of the telomeric complex in rodents: two distinct POT1 proteins protect mouse telomeres. Cell 2006; 126:6377.

15 Kibe T, Zimmermann M, de Lange T. TPP1 blocks an ATR-mediated resection mechanism at telomeres. Mol Cell 2016; 61:236-246.

16 Kim SH, Kaminker P, Campisi J. TIN2, a new regulator of telomere length in human cells. Nat Genet 1999; 23:405-412.

17 Ye JZ, Donigian JR, van Overbeek M, et al. TIN2 binds TRF1 and TRF2 simultaneously and stabilizes the TRF2 complex on telomeres. J Biol Chem 2004; 279:47264-47271.

18 Frescas D, de Lange T. Binding of TPP1 protein to TIN2 protein is required for POT $1 \mathrm{a}, \mathrm{b}$ protein-mediated telomere protection. J Biol Chem 2014; 289:24180-24187.

19 Frescas D, de Lange T. TRF2-tethered TIN2 can mediate telomere protection by TPP1/POT1. Mol Cell Biol 2014; 34:13491362.

20 Kim SH, Beausejour C, Davalos AR, Kaminker P, Heo SJ, Campisi J. TIN2 mediates functions of TRF2 at human telomeres. J Biol Chem 2004; 279:43799-43804.

21 O'Connor MS, Safari A, Xin H, Liu D, Songyang Z. A critical role for TPP1 and TIN2 interaction in high-order telomeric complex assembly. Proc Natl Acad Sci USA 2006; 103:1187411879.

22 Takai KK, Kibe T, Donigian JR, Frescas D, de Lange T. Telomere protection by TPP1/POT1 requires tethering to TIN2. Mol Cell 2011; 44:647-659.

23 Frank AK, Tran DC, Qu RW, Stohr BA, Segal DJ, Xu L. The Shelterin TIN2 subunit mediates recruitment of telomerase to telomeres. PLoS Genet 2015; 11:e1005410.

24 Ye JZ, de Lange T. TIN2 is a tankyrase 1 PARP modulator in the TRF1 telomere length control complex. Nat Genet 2004; 36:618-623. 
25 Zeng Z, Wang W, Yang Y, et al. Structural basis of selective ubiquitination of TRF1 by SCFFbx4. Dev Cell 2010; 18:214225.

26 Liu D, Safari A, O'Connor MS, et al. PTOP interacts with POT1 and regulates its localization to telomeres. Nat Cell Biol 2004; 6:673-680.

27 Holm L, Rosenstrom P. Dali server: conservation mapping in 3D. Nucleic Acids Res 2010; 38:W545-W549.

28 Imielinski M, Berger AH, Hammerman PS, et al. Mapping the hallmarks of lung adenocarcinoma with massively parallel sequencing. Cell 2012; 150:1107-1120.

29 Boora GK, Kanwar R, Kulkarni AA, et al. Exome-level comparison of primary well-differentiated neuroendocrine tumors and their cell lines. Cancer Genet 2015; 208:374-381.

30 Attwooll CL, Akpinar M, Petrini JH. The mre11 complex and the response to dysfunctional telomeres. Mol Cell Biol 2009; 29:5540-5551.

31 Deng Y, Guo X, Ferguson DO, Chang S. Multiple roles for MRE11 at uncapped telomeres. Nature 2009; 460:914-918.

32 Dimitrova N, de Lange T. Cell cycle-dependent role of MRN at dysfunctional telomeres: ATM signaling-dependent induction of nonhomologous end joining (NHEJ) in G1 and resection-mediated inhibition of NHEJ in G2. Mol Cell Biol 2009; 29:5552-5563.

33 Rai R, Zheng H, He H, et al. The function of classical and alternative non-homologous end-joining pathways in the fusion of dysfunctional telomeres. EMBO J 2010; 29:2598-2610.

34 Wang $\mathrm{M}, \mathrm{Wu}$ W, Rosidi B, Zhang L, Wang H, Iliakis G. PARP-1 and $\mathrm{Ku}$ compete for repair of DNA double strand breaks by distinct NHEJ pathways. Nucleic Acids Res 2006; 34:6170-6182.

35 Yan CT, Boboila C, Souza EK, et al. IgH class switching and translocations use a robust non-classical end-joining pathway. Nature 2007; 449:478-482.

36 Boboila C, Yan C, Wesemann DR, et al. Alternative end-joining catalyzes class switch recombination in the absence of both Ku70 and DNA ligase 4. J Exp Med 2010; 207:417-427.

37 Simsek D, Jasin M. Alternative end-joining is suppressed by the canonical NHEJ component Xrcc4-ligase IV during chromosomal translocation formation. Nat Struct Mol Biol 2010; 17:410-416.

38 Sfeir A, de Lange T. Removal of shelterin reveals the telomere end-protection problem. Science 2012; 336:593-597.

39 Chen Y, Rai R, Zhou ZR, et al. A conserved motif within RAP1 has diversified roles in telomere protection and regulation in different organisms. Nat Struct Mol Biol 2011; 18:213-
221.

40 Chen $\mathrm{C}, \mathrm{Gu} \mathrm{P}, \mathrm{Wu}$ J, et al. Structural insights into POT1TPP1 interaction and POT1 C-terminal mutations in human cancer. Nat Commun 2017; 8:14929.

41 Rice C, Shastrula PK, Kossenkov AV, et al. Structural and functional analysis of the human POT1-TPP1 telomeric complex. Nat Commun 2017; 8:14928.

42 Shi T, Bunker RD, Mattarocci S, et al. Rif1 and Rif2 shape telomere function and architecture through multivalent Rap1 interactions. Cell 2013; 153:1340-1353.

43 Spengler D. The protein kinase Aurora C phosphorylates TRF2. Cell Cycle 2007; 6:2579-2580.

44 Fairall L, Chapman L, Moss H, de Lange T, Rhodes D. Structure of the TRFH dimerization domain of the human telomeric proteins TRF1 and TRF2. Mol Cell 2001; 8:351-361.

45 Broccoli D, Smogorzewska A, Chong L, de Lange T. Human telomeres contain two distinct Myb-related proteins, TRF1 and TRF2. Nat Genet 1997; 17:231-235.

46 Benarroch-Popivker D, Pisano S, Mendez-Bermudez A, et al. TRF2-mediated control of telomere DNA topology as a mechanism for chromosome-end protection. Mol Cell 2016; 61:274-286.

47 Chen LY, Zhang Y, Zhang Q, et al. Mitochondrial localization of telomeric protein TIN2 links telomere regulation to metabolic control. Mol Cell 2012; 47:839-850.

48 Lam YC, Akhter S, Gu P, et al. SNMIB/Apollo protects leading-strand telomeres against NHEJ-mediated repair. EMBO J 2010; 29:2230-2241.

49 Otwinowski Z, Minor W. Processing of X-ray Diffraction Data Collected in Oscillation Mode Methods in Enzymology. New York: Academic Press, 1997:307-326.

50 Vonrhein C, Blanc E, Roversi P, Bricogne G. Automated structure solution with autoSHARP. Methods Mol Biol 2007; 364:215-230.

51 Adams PD, Grosse-Kunstleve RW, Hung LW, et al. PHENIX: building new software for automated crystallographic structure determination. Acta Crystallogr D Biol Crystallogr 2002; 58:1948-1954.

52 Emsley P, Cowtan K. Coot: model-building tools for molecular graphics. Acta Crystallogr D Biol Crystallogr 2004; 60:2126-2132.

53 Takai H, Smogorzewska A, de Lange T. DNA damage foci at dysfunctional telomeres. Curr Biol 2003; 13:1549-1556.

54 Rai R, Chang S. Probing the telomere damage response. Methods Mol Biol 2017; 1587:133-138.

(Supplementary information is linked to the online version of the paper on the Cell Research website.) 